1 Local protein synthesis of mtIF3 regulates mitochondrial translation for axonal

\title{
2 development
}

3 Soyeon Lee ${ }^{1,2, \dagger}$, Dongkeun Park ${ }^{1,2, \dagger}$, Chunghun Lim ${ }^{1, *}$, Jae-Ick Kim ${ }^{1, *}$, and Kyung-Tai Min ${ }^{1,2,3, *}$

$4 \quad{ }^{1}$ Department of Biological Sciences, Ulsan National Institute of Science and Technology (UNIST),

5 Ulsan 44919, Republic of Korea

$6 \quad{ }^{2}$ National Creative Research Initiative Center for Proteostasis, Ulsan National Institute of Science and

7 Technology (UNIST), Ulsan 44919, Republic of Korea

$8 \quad{ }^{3}$ Deceased July 23, 2020

$9{ }^{\dagger}$ These authors contributed equally to this work.

$10 \quad{ }^{*}$ Correspondence: C.L. (clim@unist.ac.kr) or J.-I.K. (jikim220@unist.ac.kr) 


\section{Abstract}

13 Mitochondrial initiation factor 3 (mtIF3) binds to and dissociates mitochondrial ribosomes. The mtIF3-

14 small subunit complex then recruits mtIF2, mRNA, and N-formylmethionine-tRNA to initiate

15 mitochondrial translation. Intriguingly, transcripts of the nuclear-encoded mtIF3 gene have been shown

16 present in axonal growth cones; however, the biological function of this compartmentalization remains

17 largely unknown. Here, we demonstrate that brain-derived neurotrophic factor (BDNF) induces local

18 translation of mtIF3 mRNA in axonal growth cones. Subsequently, mtIF3 protein is translocated into

19 axonal mitochondria and promotes mitochondrial translation as assessed by our newly developed

20 bimolecular fluorescence complementation sensor for the assembly of mitochondrial ribosomes. We

21 further show that BDNF-induced axonal growth requires mtIF3-dependent mitochondrial translation in

22 axons. These findings provide new insight into how neurons adaptively control mitochondrial

23 physiology and axonal development via local mtIF3 translation. 


\section{Introduction}

Mitochondrial oxidative phosphorylation (OXPHOS) complexes primarily generate ATP essential for cellular function in neuronal cell bodies and neurites. In fact, mitochondria are transported to axons and produce local energy for axon branching, growth cone formation, and axon growth (Rangaraju, Lauterbach, \& Schuman, 2019; Spillane, Ketschek, Merianda, Twiss, \& Gallo, 2013; Vaarmann et al., 2016). The localized mitochondria also play a significant role in facilitating axonal regeneration after injury (Han, Baig, \& Hammarlund, 2016; Lee, Wang, Hwang, Namgung, \& Min, 2019; Zhou et al., 2016). Thus, rapid ATP synthesis in response to local energy demand is likely crucial, particularly for polarized neuronal function.

Although active mitochondrial transport to axonal tip has been shown to support local energy needs (Saxton \& Hollenbeck, 2012; Sheng, 2017), this may not be sufficient to explain how neurons adaptively regulate mitochondrial function in axons (Niescier, Kwak, Joo, Chang, \& Min, 2016). Hence, we reason that additional mechanisms, such as local synthesis of mitochondrial proteins, should contribute to the functional control of axonal mitochondria. Most mitochondrial genes are nuclearencoded, and once transcribed, their mRNA translation generally occurs in the cell bodies. On the other hand, previous studies have revealed that transcripts of the nuclear-encoded mitochondrial genes can be locally translated in axons (A. Aschrafi et al., 2016; Gale, Aschrafi, Gioio, \& Kaplan, 2018; Kaplan, Gioio, Hillefors, \& Aschrafi, 2009; Kuzniewska et al., 2020; Shigeoka et al., 2016). Nonetheless, it is still elusive whether any local synthesis of the nuclear-encoded mitochondrial proteins governs mitochondrial function in axons.

In mammalian cells, mitochondria have only two mitochondrial translation initiation factors, mtIF2 and mtIF3 (Smits, Smeitink, \& van den Heuvel, 2010). Interestingly, translatome analyses have revealed mtIF3 translation in axon growth cone (Shigeoka et al., 2016), suggesting a possible role of local mtIF3 synthesis in regulating axonal mitochondrial translation. mtIF3 regulates the dynamics of ribosome association on mitochondrial mRNAs. mtIF3 catalyzes the dissociation of mitochondrial ribosomes (mitoribosomes) into large and small subunits while blocking any premature binding of the large subunit (Christian \& Spremulli, 2009; Koc \& Spremulli, 2002). mtIF2 and N-formylmethionine-tRNA bind 
weakly to the small subunit in the absence of mRNA, but mtIF3 facilitates mRNA binding to the small subunit so that a start codon can be correctly positioned at P-site (Christian \& Spremulli, 2009; Smits et al., 2010).

Given the critical role of mtIF3 in mitochondrial translation initiation (Rudler et al., 2019), it is plausible that locally synthesized mtIF3 may regulate mitochondrial translation in developing axons to support ATP synthesis and relevant physiology. Studies on mitochondrial translation in live cells, however, have been hampered by a lack of appropriate tools. Here, we have developed a molecular sensor that visualizes mitochondrial translation activity using the bimolecular fluorescence complementation $(\mathrm{BiFC})$ between a specific pair of mitoribosome proteins. In conjunction with additional transgenic reporters for functional imaging, this new tool has led us to test the hypothesis above and validate the significance of local mtIF3 translation in mitochondrial physiology and axonal growth.

\section{Results}

\section{BDNF induces local protein synthesis of mtIF3 in axon growth cone}

We first confirmed that mtIF3 mRNAs were present in both cell bodies and axons of primary hippocampal neurons (Figure 1A), consistent with a previous report (Shigeoka et al., 2016). To examine whether locally translated mtIF3 proteins translocate into mitochondria, we generated a transgene that expresses fluorescent mtIF3 proteins fused to the photo-convertible Dendra2 along with N-terminal palmitoylation sequence (mtIF3-Dendra2) (Lee et al., 2019; Wang et al., 2016) and mtIF3 untranslated regions (UTRs) (5'UTR ${ }_{\mathrm{mtIF3}}-\mathrm{mtIF}$-Dendra2-3'UTR ${ }_{\mathrm{mtIF3}}$ ). As expected, the coding sequence (CDS) of mtIF3 led to mitochondrial localization of mtIF3-Dendra2 fusion likely due to its mitochondrial targeting sequence (Figure 1-figure supplement 1). The fluorescent mtIF3-Dendra2 proteins in axons were irreversibly photo-switched from green to red using $405 \mathrm{~nm}$ illumination, and then newly translated mtIF3-Dendra2 proteins with green fluorescence were measured by analyzing time-lapse images taken every 5 minutes for 90 minutes. Several studies have suggested that many nuclear-encoded mitochondrial proteins might be synthesized in response to local energy demand (Gale et al., 2018; 
Kaplan et al., 2009; Kuzniewska et al., 2020; Shigeoka et al., 2016). This prompted us to examine whether BDNF treatment in axonal growth cone enhances the local translation of the mtIF3-Dendra2 fusion reporter. Indeed, kymograph analyses revealed that BDNF treatment elevated the newly synthesized mtIF3-Dendra2 signals in axonal mitochondria (Figure 1B-C). A translation inhibitor, anisomycin, blocked the de novo synthesis of reporter proteins, validating that BDNF treatment triggers the local synthesis of mtIF3 proteins in axons and promotes their translocation into axonal mitochondria. Interestingly, mtIF3 3'UTR contains a consensus motif (CTCCCATC) shared by axon-enriched mRNAs (Shigeoka et al., 2016). We thus generated two additional translation reporters encoding the

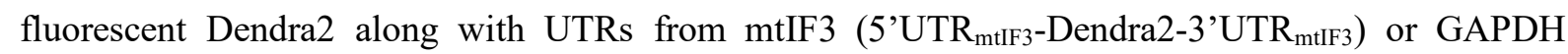
$\left(5^{\prime} \mathrm{UTR}_{\mathrm{GAPDH}}-\mathrm{Dendra2-3}{ }^{\prime} \mathrm{UTR}_{\mathrm{GAPDH}}\right)$. BDNF treatment gradually increased the fluorescence of newly synthesized Dendra2 from the mtIF3 UTR reporter at the axonal tip, whereas anisomycin treatment suppressed it (Figure 1D-E). We detected no significant changes in the fluorescence from the control GAPDH UTR reporter upon BDNF treatment. Together, these results indicate that mtIF3 proteins are locally synthesized in axon growth cones and translocate into mitochondria in response to BDNF. In addition, mtIF3 UTRs likely support the axonal transport of mtIF3 mRNAs and their BDNF-induced translation in axons, regardless of mitochondrial targeting of the translation products.

\section{Mito-riboBiFC detects translation-dependent assembly of mitoribosomes}

We hypothesized that BDNF-induced local translation of mtIF3 proteins might be involved in regulating mitochondrial translation in developing axon tip. To overcome possible limitations in the biochemical assessment of mitochondrial translation in axons, we devised a new strategy to visualize mitochondrial translation in live cells using $\mathrm{BiFC}(\mathrm{Hu}, \mathrm{Chinenov,} \mathrm{\&} \mathrm{Kerppola,} \mathrm{2002)} \mathrm{which} \mathrm{was}$ previously used for the visualization of cytoplasmic ribosomal subunit joining (Al-Jubran et al., 2013). This was based on the physical proximity of mitochondrial ribosomal protein L2 (MRPL2) and mitochondrial ribosomal protein S6 (MRPS6) at the inter-subunit bridge of 55S mitoribosome (Amunts, Brown, Toots, Scheres, \& Ramakrishnan, 2015) (Figure 2A). We took advantage of this adjacent localization of the two MRPs as a BiFC pair to visualize mitoribosome assembly during translation. In 
detail, we split a fluorescent protein mVenus into N-terminal (VN, 1-172 amino acids) and C-terminal fragments (VC, 155-238 amino acids). Then we fused these mVenus fragments to the C-termini of MRPS6 (S6-VN) and MRPL2 (L2-VC), respectively. The co-expression of S6-VN and L2-VC in Neuro2A cells generated mVenus fluorescent signals exclusively in mitochondria (Figure 2B). On the other hand, another pair of MRPs positioned distantly from each other in mitoribosomes showed relatively weak fluorescent signals (Figure 2A-D, MRPS16 and MRPL50) even though the latter BiFC pair were expressed comparably to S6-VN and L2-VC (Figure 2B-D).

Unlike cytoplasmic ribosomes, two mitoribosome subunits could be assembled in the absence of translating mRNAs (Smits et al., 2010). We thus asked if the BiFC signals from the S6-VN and L2-VC pair would depend on the translation of mitochondrial mRNAs. When we treated puromycin to dissociate translating ribosome subunits from mRNAs, the BiFC signals were reduced only by $8 \%$ compared to the vehicle control (Figure 2E and F), indicating the mRNA-independent assembly of the BiFC pair. The average cytoplasmic translation rate is six amino acids per second (Ingolia, Lareau, \& Weissman, 2011), and the longest transcript mt-ND5 mRNA is $1824 \mathrm{bp}$. In contrast, the fluorescence signals from the BiFC pair become detectable 10 minutes after complementation (Robida \& Kerppola, 2009). Considering that the translation of individual mitochondrial mRNAs could be completed in less than 2 minutes, it is likely that the translating mitoribosome dissociates from mRNAs before the chromophore maturation. We thus reasoned that the stabilization of translating mitoribosome would better visualize their mRNA-dependent emission of the complemented fluorescence signals. Indeed, chloramphenicol (CA), an inhibitor of the peptide bond formation at mitoribosome E-site, markedly increased the BiFC signals 60 minutes after treatment, whereas puromycin pre-treatment blocked the CA effects (Figure 2E-F). Accordingly, we concluded that CA-induced BiFC signals would represent actively translating mitoribosome and designated our new tool for visualizing mitochondrial translation as mito-riboBiFC (Figure 2-figure supplement 1).

\section{Locally synthesized mtIF3 promotes mitochondrial translation in axon growth cone}

To test whether locally synthesized mtIF3 facilitates mitochondrial translation in the axon growth 
cone, we manipulated mtIF3 expression by transient transfections and examined their effects on the mito-riboBiFC signals in axons. We first confirmed mtIF3 depletion using short hairpin RNA (shRNA) or transgenic mtIF3 overexpression in NIH/3T3 cells (Figure 3-figure supplement 1). Next, we cultured hippocampal neurons on a microfluidic device to separate axons from cell bodies (Taylor et al., 2005) and treated all the drugs only in the axonal channel to induce or block local translation (Figure 3A-B). The degree of mitochondrial translation was subsequently quantified using CA-induced changes in the intensity of mito-riboBiFC (Figure 2-figure supplement 1). Notably, neurons expressing control shRNA exhibited BDNF-induced BiFC signals in the axon growth cone. However, the treatment of a translation inhibitor cycloheximide (CHX) completely blocked the increment of BiFC signals upon BDNF treatment, indicating that BDNF-induced local protein synthesis promotes mitochondrial translation. Importantly, mtIF3 depletion abolished the BDNF-induced mito-riboBiFC signals (Figure 3C-D), suggesting that locally synthesized mtIF3 is necessary for facilitating mitochondrial translation upon BDNF treatment.

To assess whether mtIF3 overexpression elevates the mitochondrial translation in the absence of BDNF, we transfected primary hippocampal neurons with a mtIF3 overexpression vector at DIV2 and measured any change in the mito-riboBiFC signals. Neither the baseline nor BDNF-induced mitoriboBiFC signals were significantly affected by mtIF3 overexpression under our experimental conditions. Considering that our transgene for mtIF3 overexpression included mtIF3 UTRs, we reason that local synthesis of mtIF3 would be tightly regulated at post-transcript levels, and the axonal abundance of mtIF3 mRNAs may not be limiting for BDNF-induced mitochondrial translation. Together, these results support that BDNF-induced local protein synthesis of mtIF3 leads to enhanced mitochondrial translation in the axon growth cone.

\section{mtIF3-dependent mitochondrial translation elevates ATP generation in growing axons}

Next, we questioned whether locally translated mtIF3 would control mitochondrial physiology in developing axons. To this end, we employed mito-ATeam1.03, a genetically encoded FRET sensor for mitochondrial ATP (Imamura et al., 2009). CA treatment to primary hippocampal neurons expressing 
mito-ATeam 1.03 reduced the intensity of the FRET signals, indicating that mitochondrial ATP generation requires mitochondrial translation (Figure 4-figure supplement 1). We further found that BDNF treatment elevated mitochondrial ATP levels in the axon growth cone, whereas blocking local translation by CHX nullified the BDNF effects (Figure 4A-B). mtIF3 depletion also blunted BDNFinduced increase in mitochondrial ATP levels, yet it negligibly affected the baseline ATP levels (Figure 4A-B). We observed no significant effects of mtIF3 overexpression on mitochondrial ATP levels in axons regardless of BDNF treatment (Figure 4C-D), consistent with mtIF3 effects on mitochondrial translation in axons. These results support our model that BDNF-induced local synthesis of mtIF3 promotes mitochondrial translation and elevates ATP generation in axonal mitochondria, thereby fulfilling local energy demand in the developing axons.

\section{Axonal development requires mtIF3-dependent mitochondrial translation in growing axons}

To determine whether local mitochondrial translation indeed impacts axonal growth, we applied CA to either cell bodies or axons of primary hippocampal neurons cultured in a microfluidic device (Figure 5A). BDNF was subsequently added to the axonal chamber, and BDNF-induced axon growth was quantified accordingly. We found that selective inhibition of mitochondrial translation in axons, but not in cell bodies, suppressed BDNF-induced axon extensions (Figure 5B-C). These data demonstrate that rapid axon extension by this trophic factor requires local mitochondrial translation in axons. Given that locally synthesized mtIF3 regulates the mitochondrial translation in axons, we reasoned that mtIF3 depletion would impair axonal extension. Indeed, transient overexpression of mtIF3 shRNA remarkably shortened axonal length compared to control shRNA (Figure 5D-E). Moreover, mtIF3 depletion silenced BDNF effects on axon development. We observed that mtIF3 overexpression negligibly affected axon growth regardless of BDNF treatment (Figure 5-figure supplement 1), consistent with its lack of any significant effects on mitochondrial translation and ATP generation in axons. Together, our findings validate that mtIF3-dependent mitochondrial translation in axons plays a critical role in axonal development. 


\section{Discussion}

Local protein synthesis is a distinctive feature in neurons that have highly polarized neurites, which is indispensable for the maintenance of axonal or dendritic structures and functions such as neurite development, the guidance of growth cone, synaptic transmission, synaptic plasticity, branch formation, and regeneration (Hafner, Donlin-Asp, Leitch, Herzog, \& Schuman, 2019; Jung, Yoon, \& Holt, 2012; Shigeoka et al., 2019). The gene ontology analyses revealed high enrichment of synaptic proteins, cytoskeletal proteins, and ribosomal proteins in axonal translatome (Gumy et al., 2011; Shigeoka et al., 2016). Interestingly, it has been also identified that transcripts of nuclear-encoded mitochondrial proteins are abundant in developing and mature axons (A. Aschrafi et al., 2016; Gale et al., 2018; Kaplan et al., 2009; Kuzniewska et al., 2020; Shigeoka et al., 2016), implicating their local translation in sustaining mitochondrial function and axonal viability. Nonetheless, only a few studies have documented that local translation of nuclear-encoded mitochondrial proteins can affect mitochondrial function and axonal survival (Armaz Aschrafi, Natera-Naranjo, Gioio, \& Kaplan, 2010; Hillefors, Gioio, Mameza, \& Kaplan, 2007; Natera-Naranjo et al., 2012; Yoon et al., 2012).

Here, we demonstrate that nuclear-encoded mtIF3 is locally translated in developing axons, thereby promoting axonal mitochondrial translation as assessed by our newly developed mito-riboBiFC sensor. Many studies have demonstrated that stationary mitochondria in axons fuel spatially restricted boundaries (Rangaraju et al., 2019; Spillane et al., 2013), but what remains unsolved is how these stationary mitochondria are supported and maintained in the long-term. Our results suggest that mitochondrial proteins may be replenished by enhanced mitochondrial translation via local protein synthesis in axons. We observed that the mtIF3 depletion cancels out the upregulation of mitochondrial translation and ATP production upon BDNF stimulation. Lack of this local translation and adaptive control of mitochondrial function limits axonal development, validating its critical role in neuronal physiology. However, we also observed that the overexpression of mtIF3 per se did not affect mitochondrial functions. It has been recently shown that mitochondrial translation is synchronized and unidirectionally controlled by cytosolic translation (Couvillion, Soto, Shipkovenska, \& Churchman, 2016). Our observation consistently implicates that enhanced mitochondrial functions for local energy 
214 demanding are accomplished by not the mitochondrial translation alone but the simultaneous cytosolic

215 translation. Therefore, our findings suggest that local translation in axons can be a crucial mechanism

216 by which mitochondrial translation is regulated in mammalian neurons.

217 In the past decade, much effort has been made to develop the tools for measuring or observing 218 mitochondrial translation. For instance, biochemical detection of newly synthesized mitochondrial 219 proteins has been widely used for studying mitochondrial translation (Barsh et al., 2015; ChatenayLapointe \& Shadel, 2011; Park, Lee, \& Min, 2020; Richter-Dennerlein et al., 2016; Richter, Lahtinen, Marttinen, Suomi, \& Battersby, 2015). However, a lack of appropriate imaging tools for mitochondrial translation has hindered assessing this subcellular event at single-cell levels. A recent study visualized mitochondrial translation using a non-canonical amino acid labeling in situ (Estell, Stamatidou, ElMesseiry, \& Hamilton, 2017). This method allows the detection of mitochondrial translation at a singlecell resolution, but its application is limited to fixed cells. Our study developed a new method designated as mito-riboBiFC to monitor mitochondrial translation in live cells. Mito-riboBiFC enables us to investigate mitochondrial translation on distinct spatiotemporal scales. Accordingly, it will be of great interest to determine how mitochondrial translation is regulated depending on their subcellular location or mitochondrial dynamics, especially in neurons where subcellular environment and energetic needs are spatially distinct.

Nonetheless, mito-riboBiFC has some limitations that should be improved in the future. These include relatively slow maturation kinetics of the mito-riboBiFC. Mitochondria are highly dynamic and heterogeneous in terms of their transport, membrane potential, and biogenesis. These mitochondrial events can occur on a relatively short timescale (e.g., a few seconds or minutes), compared to the folding and maturation time of the BiFC complex (Rose, Briddon, \& Holliday, 2010). The employment of a new chromophore in BiFC imaging should improve the current temporal resolution of our mitoriboBiFC, better visualizing the rapid change in mitochondrial translation according to diverse mitochondrial dynamics.

In conclusion, our results provide new insights into understanding the adaptive regulation of mitochondrial physiology via local protein synthesis of a nuclear-encoded mitochondrial translation 
factor during axonal development. New imaging tools for the mitochondrial function should further

242 dissect the molecular mechanisms underlying the spatiotemporal control of mitochondria physiology and hint at novel therapeutic strategies to treat relevant neurodevelopmental diseases.

\section{Materials and methods}

$\underline{\text { Animals }}$

Pregnant mice (C57BL/6J, Hyochang Science, Korea) were used for primary hippocampal neuron culture. All experimental procedures were conducted in accordance with protocols approved by Institutional Animal Care and Use Committee of Ulsan National Institute of Science and Technology (UNIST).

Cell culture

Primary hippocampal neurons

Primary hippocampal neuron culture was processed as follows. In brief, hippocampi were dissected from E18 mouse embryos and they were washed with HBSS (Invitrogen). Hippocampi were digested by $0.025 \%$ trypsin (Invitrogen) and washed with trituration media ( $90 \%$ of Dulbecco Modified Eagle Medium and $10 \%$ fetal bovine serum, Invitrogen). Dissociated cells were seeded onto $50 \mu \mathrm{g} / \mathrm{ml}$ of polyD-lysine (Sigma) coated culture dishes or coverslips. After settlement of cells, neurons were maintained with neuronal culture media, which consists of Neurobasal media, GlutaMax, B27, and penicillinstreptomycin (Invitrogen). Neurons were transfected with lipofectamine 2000 (Invitrogen).

\section{Cell lines}

Neuro2A cell line was used for mito-riboBiFC experiments and purchased from ATCC. Neuro2A cells were maintained in culture media, which consists of Dulbecco Modified Eagle Medium and 10\% fetal bovine serum, and 1\% penicillin-streptomycin (Invitrogen). Using mycoplasma detection kit (Takara, 6601), we confirmed no contamination in Neuro2A cell line. PEI (Polysciences, 23966-1) or lipofectamine 2000 (Invitrogen) were used for transfecting constructs into Neuro2A cells. NIH/3T3 was 
purchased from ATCC and used for the modulation of mtIF3 expression. Cells were maintained in culture media, which consists of Dulbecco Modified Eagle Medium and 10\% calf serum, and 1\% penicillin-streptomycin (Invitrogen). Metafectene (Biontex) was used for the transfection of mtIF3 constructs.

\section{$\underline{\text { Separation of cell bodies and axons }}$}

To isolate lysate of cell bodies and axons separately, neurons were seeded on the 6-well inserts with 3 $\mu \mathrm{m}$ pore size (SPL Life Sciences). Samples of cell bodies and axons were collected by scrapping the upper and bottom side of inserts. To treat cell bodies or axons separately with drugs, neurons were placed on microfluidic devices (Xona Microfluidics). Microfluidic devices were attached to glass bottom dish (In Vitro Scientific, D60-30-1.5) for live cell imaging or $22 \mathrm{~mm}$ square coverslips (Globe Scientific, 1404-15) for fixed samples. $30 \mathrm{ng} / \mathrm{ml}$ of BDNF (Sigma), $50 \mu \mathrm{g} / \mathrm{ml}$ of chloramphenicol (Sigma), $20 \mu \mathrm{M}$ of anisomycin (Sigma), and $100 \mu \mathrm{g} / \mathrm{ml}$ of cycloheximide (Sigma) were used for drugs treatment.

\section{Vector preparation}

For local protein synthesis assay, $p D e n d r a 2-C$ vector (Evrogen) was modified: 5 'UTR of mtIF3-2xPalDendra2-3'UTR of mtIF3, 5'UTR of mtIF3-CDS of mtIF3-2xPal-Dendra2-3'UTR of mtIF3, and 5'UTR of GAPDH-2xPal-Dendra2-3'UTR of GAPDH. To block the effect of diffusion, two repeats of palmitoylation sequence was added. For mito-riboBiFC assay, $p c D N A 6 / V 5-H i s A$ (Invitrogen) plasmid was modified: Neuro2A cDNA sequence of Mouse Mrps6 (NM_080456.1), Mrpl2 (NM_025302.4), Mrps 16 (NM_025440.3), and Mrpl50 (NM_178603.4) were used to generate MRPS6-VN172, MRPL2VC155, MRPS16-VN172, and MRPL50-VC155 constructs. VC was fused to MRPL2 and MRPL50 by linker peptides: GSKQKVMNH. MRPS6 and MRPS16 were fused to VN by linker peptides: GSRSIAT. For the modulation of mtIF3 expression, AAV-shRNA-ctrl (Addgene, \#85741), and pcDNA6/V5-HisA plasmid was modified: pAAV2-Control-shRNA-TagRFP657, pAAV2-mtIF3-shRNA-TagRFP657, pcDNA6-5'UTR of mtIF3-3xFLAG-P2A-TagRFP657-3'UTR of mtIF3, and pcDNA6-5'UTR of mtIF3- 
CDS of mtIF3-3xFLAG-P2A-TagRFP657-3'UTR of mtIF3.

\section{Confocal microscopy and image analysis}

298 All the images were taken using a confocal microscope (Zeiss LSM 780). Live cell imaging was

performed in a live cell chamber that was maintained at $37^{\circ} \mathrm{C}$ and $5 \% \mathrm{CO}_{2}$ by heating instrument.

Definite Focus z-correction hardware was used to maintain the z-axis during the time lapse image. intensity was quantified by ImageJ (NIH).

$\underline{\text { Local protein synthesis assay }}$

For local mRNA translation assay, Dendra2 fluorescence protein was conjugated with UTRs of mtIF3 or GAPDH: 5'UTR of mtIF3-Palmitoylation sequence-Dendra2-3'UTR of mtIF3, 5'UTR of mtIF3CDS of mtIF3-Palmitoylation sequence-Dendra2-3'UTR of mtIF3, and 5'UTR of GAPDHwith these vectors at DIV3 by using Lipofectamine 2000 (Invitrogen). 24 hours after transfection, protein synthesis assay was performed. Existing fluorescence of dendra2 (green) was photoconverted into red fluorescence with $405 \mathrm{~nm}$ laser for 10 seconds and newly synthesized green signals were measured for 90 minutes with 5 minutes time lapse image. Protein synthesis inhibitor, anisomycin (20 $\mu \mathrm{M}$, Sigma) was used to confirm that the increased green signal was from de novo protein synthesis.

Live cell imaging

Before mito-riboBiFC imaging in Neuro2A cells, culture medium was replaced and cells were incubated for 30 minutes. $20 \mu \mathrm{M}$ of puromycin (Sigma) was applied for 10 minutes. After puromycin treatment, was also transfected. Images were acquired using 458, 514nm lasers. 
attached on glass bottom dishes. Neurons were transfected with shRNAs at DIV1 and then transfected with overexpression vectors at DIV2. At DIV3, images were acquired using 458, 514, and $633 \mathrm{~nm}$ lasers. To fix mitochondrial ribosomes, chloramphenicol was treated for 90 minutes. BDNF or cycloheximide were also treated simultaneously to induce or block local translation and images were taken after 90 minutes. Fluorescent intensity was measured from five mitochondria at the end of axons. The ratio of before and after drug treatment was averaged to measure the degree of mitochondrial translation. For mitochondrial ATP imaging, primary hippocampal neurons were transfected with genetically encoded FRET-based ATP indicator for mitochondria, mito-ATeam1.03, at DIV1 for mtIF3 knockdown study or at DIV2 for mtIF3 overexpression study by using Lipofectamine 2000 (Invitrogen). Images were taken at emission of $475 \mathrm{~nm}$ and $527 \mathrm{~nm}$ with a $405 \mathrm{~nm}$ excitation laser. BDNF or cycloheximide were applied to axonal chamber for 90 minutes. The increased ratio of FRET to CFP before and after drug treatment was calculated.

For mtIF3 localization determination imaging, annotated vector was transfected using PEI in Neuro2A. 250nM of MitoTracker ${ }^{\mathrm{TM}}$ Deep Red FM (Invitrogen, M22426) was added to cells and images were acquired after 10 minutes. Images were acquired using 488, $633 \mathrm{~nm}$ lasers.

Western blotting

Cells were lysed by using RIPA buffer (150 mM sodium chloride, 1\% Triton X-100, 0.5\% sodium deoxycholate and $0.1 \%$ sodium dodecyl sulfate). Proteins were separated by SDS-PAGE and transferred to PVDF membranes (Millipore). Membranes were blocked with 5\% skim milk in TBST (10 mM Tris, $150 \mathrm{mM} \mathrm{NaCl}, 0.5 \%$ Tween 20) for 30 minutes. For immunoblotting, antibodies against mtIF3 (Sigma, Rad). 
Total RNA of cell body and axon fractionations was isolated with PicoPure RNA isolation kit (Applied

Biosystems). 200 ng of RNA was subjected to RT-PCR by using High Capacity RNA-to-cDNA kit (Life

Technologies). The primers used for PCR:

forward 5'-GAGAGCAGATCCACCAGGAG-3' and

reverse 5'-CTGTTTCCGTCGTCGTCTTT-3' for mtIF3;

forward 5'-ACCAACTGGGACGACATGGAGAAGA-3' and

reverse 5'-CGTTGCCAATAGTGATGACCTGGCC-3' for $\beta$-actin;

forward 5'-GGACGACATGGAGAAGATCTGGCAC-3' and

reverse 5'-CCGGACACCGGAACCGCTCATTG-3' for $\gamma$-actin.

Immunostaining

For primary hippocampal neurons and NIH/3T3 cells, cells were rinsed with PBS and fixed with 4\% PFA for 10 minutes. Cell were permeabilized with PBST (PBS with $0.2 \%$ Triton X-100) for 10 minutes and blocked with $1 \%$ BSA in PBST for 30 minutes. Primary antibodies were incubated at $4^{\circ} \mathrm{C}$ for polyclonal), and MTCO1 (Abcam, ab203912, monoclonal) were used for immunostaining. The cells were washed three times with PBS and incubated with Alexa Fluor secondary antibodies (Invitrogen). Then these cells were washed three times with PBS and coverslips were mounted on slide glasses. Images were taken by using LSM780 confocal microscopy. For Neuro2A, cells were washed with icecold PBS followed by fixation using 4\% PFA/sucrose for 15 minutes. After 3 times washing with PBS, cells were permeabilized with PBST (PBS with $0.5 \%$ Triton X-100) for 15 minutes and blocked with temperature. Cells were washed three times with PBS and coverslips were mounted on slide glasses. 
Antibodies against GFP (Abcam, ab 6556, polyclonal) and MT-CO1 (Abcam, ab14705, monoclonal) were used for immunostaining.

\section{$\underline{\text { Statistical analysis }}$}

Statistical analyses were performed by using Prism software (GraphPad Software) or R (version 3.6.1) with ARTool library (Kay \& Wobbrock, 2016; Wobbrock, Findlater, Gergle, \& Higgins, 2011). All the values were presented with mean \pm SEM. Shapiro-Wilk test for normality $(P<0.05)$ or Brown-Forsythe test for equal variance $(P<0.05)$ were used to determine the statistical analysis for each dataset. Ordinary two-way ANOVA with Dunnett's test (repeated measure) or Tukey's test (non-repeated measure), aligned ranks transformation ANOVA with Wilcoxon signed-rank test (repeated measure) or Wilcoxon rank-sum (non-repeated measure) were used to determine statistical differences between the groups. P $<0.05$ was considered as statistically significant. $* P<0.05$, $* * P<0.01, * * * P<0.001, * * * * P<0.0001$.

\section{Acknowledgements}

We wish to dedicate this work to the life and career of our beloved mentor and colleague, Dr. KyungTai Min, who passed away recently. This work was supported by Basic Science Research Program through the National Research Foundation of Korea (NRF) funded by the Ministry of Science and ICT (2016R1A3B1905982 to K.M., 2020R1A2C1005492 to J.-I.K.). This work was also supported by a grant from the Suh Kyungbae Foundation (SUHF-17020101 to C.L.).

\section{Competing interests}

No competing interests declared

\section{Author contribution}

Conceptualization, D.P. and K.M.; Methodology, S.L., D.P., C.L., J.-I.K. and K.M.; Investigation, S.L. and D.P.; Interpretation of Data, S.L., D.P., C.L., J.-I.K. and K.M.; Writing - Original Draft, S.L., D.P. 
and K.M.; Writing - Review \& Editing, S.L., D.P., C.L. and J.-I.K.; Funding Acquisition, C.L., J.-I.K. and K.M.; Supervision, C.L., J.-I.K. and K.M.

\section{References}

Al-Jubran, K., Wen, J., Abdullahi, A., Roy Chaudhury, S., Li, M., Ramanathan, P., . . Brogna, S. (2013). Visualization of the joining of ribosomal subunits reveals the presence of $80 \mathrm{~S}$ ribosomes in the nucleus. $R N A, 19(12), 1669-1683$. doi:10.1261/rna.038356.113

Amunts, A., Brown, A., Toots, J., Scheres, S. H. W., \& Ramakrishnan, V. (2015). The structure of the human mitochondrial ribosome. Science, 348(6230), 95-98. doi:10.1126/science.aaa1193

Aschrafi, A., Kar, A. N., Gale, J. R., Elkahloun, A. G., Vargas, J. N., Sales, N., . . Kaplan, B. B. (2016). A heterogeneous population of nuclear-encoded mitochondrial mRNAs is present in the axons of primary sympathetic neurons. Mitochondrion, 30, 18-23. doi:10.1016/j.mito.2016.06.002

Aschrafi, A., Natera-Naranjo, O., Gioio, A. E., \& Kaplan, B. B. (2010). Regulation of axonal trafficking of cytochrome c oxidase IV mRNA. Molecular and Cellular Neuroscience, 43(4), 422-430.

Barsh, G. S., Lagouge, M., Mourier, A., Lee, H. J., Spåhr, H., Wai, T., . . Larsson, N.-G. (2015). SLIRP Regulates the Rate of Mitochondrial Protein Synthesis and Protects LRPPRC from Degradation. PLOS Genetics, 11(8), e1005423. doi:10.1371/journal.pgen.1005423

Chatenay-Lapointe, M., \& Shadel, G. S. (2011). Repression of mitochondrial translation, respiration and a metabolic cycle-regulated gene, SLF1, by the yeast Pumilio-family protein Puf3p. PLOS One, 6(5), e20441. doi:10.1371/journal.pone.0020441

Christian, B. E., \& Spremulli, L. L. (2009). Evidence for an Active Role of IF3mt in the Initiation of Translation in Mammalian Mitochondria. Biochemistry, 48(15), 3269-3278. doi:10.1021/bi8023493

Couvillion, M. T., Soto, I. C., Shipkovenska, G., \& Churchman, L. S. (2016). Synchronized mitochondrial and cytosolic translation programs. Nature, 533(7604), 499-503. doi:10.1038/nature18015

Estell, C., Stamatidou, E., El-Messeiry, S., \& Hamilton, A. (2017). In situ imaging of mitochondrial translation shows weak correlation with nucleoid DNA intensity and no suppression during mitosis. J Cell Sci, 13024), 4193-4199. doi:10.1242/jcs.206714

Gale, J. R., Aschrafi, A., Gioio, A. E., \& Kaplan, B. B. (2018). Nuclear-Encoded Mitochondrial mRNAs: A Powerful Force in Axonal Growth and Development. Neuroscientist, 24(2), 142-155. doi:10.1177/1073858417714225

Greber, B. J., Bieri, P., Leibundgut, M., Leitner, A., Aebersold, R., Boehringer, D., \& Ban, N. (2015). The complete structure of the $55 \mathrm{~S}$ mammalian mitochondrial ribosome. Science, 348(6232), 303308. doi:10.1126/science.aaa3872

Gumy, L. F., Yeo, G. S., Tung, Y.-C. L., Zivraj, K. H., Willis, D., Coppola, G., . . Fawcett, J. W. (2011). Transcriptome analysis of embryonic and adult sensory axons reveals changes in mRNA 
repertoire localization. Rna, 17(1), 85-98.

Hafner, A. S., Donlin-Asp, P. G., Leitch, B., Herzog, E., \& Schuman, E. M. (2019). Local protein synthesis is a ubiquitous feature of neuronal pre- and postsynaptic compartments. Science, 364(6441). doi:10.1126/science.aau3644

Han, S. M., Baig, H. S., \& Hammarlund, M. (2016). Mitochondria Localize to Injured Axons to Support Regeneration. Neuron, 92(6), 1308-1323. doi:10.1016/j.neuron.2016.11.025

Hillefors, M., Gioio, A. E., Mameza, M. G., \& Kaplan, B. B. (2007). Axon viability and mitochondrial function are dependent on local protein synthesis in sympathetic neurons. Cell Mol Neurobiol, 27(6), 701-716. doi:10.1007/s10571-007-9148-y

Hu, C.-D., Chinenov, Y., \& Kerppola, T. K. (2002). Visualization of interactions among bZIP and Rel family proteins in living cells using bimolecular fluorescence complementation. Molecular cell, 9(4), 789-798.

Imamura, H., Nhat, K. P., Togawa, H., Saito, K., lino, R., Kato-Yamada, Y., . . Noji, H. (2009). Visualization of ATP levels inside single living cells with fluorescence resonance energy transfer-based genetically encoded indicators. Proc Natl Acad Sci U S A, 106(37), 15651-15656. doi:10.1073/pnas.0904764106

Ingolia, N. T., Lareau, L. F., \& Weissman, J. S. (2011). Ribosome profiling of mouse embryonic stem cells reveals the complexity and dynamics of mammalian proteomes. Cell, 147(4), 789-802. doi:10.1016/j.cell.2011.10.002

Jung, H., Yoon, B. C., \& Holt, C. E. (2012). Axonal mRNA localization and local protein synthesis in nervous system assembly, maintenance and repair. Nat Rev Neurosci, 13(5), 308-324. doi:10.1038/nrn3210

Kaplan, B. B., Gioio, A. E., Hillefors, M., \& Aschrafi, A. (2009). Axonal protein synthesis and the regulation of local mitochondrial function. Results Probl Cell Differ, 48, 225-242. doi:10.1007/400_2009_1

Kay, M., \& Wobbrock, J. (2016). ARTool: aligned rank transform for nonparametric factorial ANOVAs. $R$ package version $0.10,2$.

Koc, E. C., \& Spremulli, L. L. (2002). Identification of mammalian mitochondrial translational initiation factor 3 and examination of its role in initiation complex formation with natural mRNAs. $J$ Biol Chem, 27738), 35541-35549. doi:10.1074/jbc.M202498200

Kuzniewska, B., Cysewski, D., Wasilewski, M., Sakowska, P., Milek, J., Kulinski, T. M., . . Dziembowska, M. (2020). Mitochondrial protein biogenesis in the synapse is supported by local translation. EMBO Rep, e48882. doi:10.15252/embr.201948882

Lee, S., Wang, W., Hwang, J., Namgung, U., \& Min, K. T. (2019). Increased ER-mitochondria tethering promotes axon regeneration. Proc Natl Acad Sci U S A, 116(32), 16074-16079. doi:10.1073/pnas.1818830116

Natera-Naranjo, O., Kar, A. N., Aschrafi, A., Gervasi, N. M., Macgibeny, M. A., Gioio, A. E., \& Kaplan, B. B. (2012). Local translation of ATP synthase subunit 9 mRNA alters ATP levels and the production of ROS in the axon. Molecular and Cellular Neuroscience, 49(3), 263-270. 
Niescier, R. F., Kwak, S. K., Joo, S. H., Chang, K. T., \& Min, K. T. (2016). Dynamics of Mitochondrial Transport in Axons. Front Cell Neurosci, 10, 123. doi:10.3389/fncel.2016.00123

Park, D., Lee, S., \& Min, K.-T. (2020). Techniques for investigating mitochondrial gene expression. BMB Reports, 53(1), 3-9. doi:10.5483/BMBRep.2020.53.1.272

Rangaraju, V., Lauterbach, M., \& Schuman, E. M. (2019). Spatially Stable Mitochondrial Compartments Fuel Local Translation during Plasticity. Cell, 176(1-2), 73-84 e15. doi:10.1016/j.cell.2018.12.013

Richter-Dennerlein, R., Oeljeklaus, S., Lorenzi, I., Ronsor, C., Bareth, B., Schendzielorz, A. B., . . . Dennerlein, S. (2016). Mitochondrial Protein Synthesis Adapts to Influx of Nuclear-Encoded Protein. Cell, 167(2), 471-483 e410. doi:10.1016/j.cell.2016.09.003

Richter, U., Lahtinen, T., Marttinen, P., Suomi, F., \& Battersby, B. J. (2015). Quality control of mitochondrial protein synthesis is required for membrane integrity and cell fitness. Journal of Cell Biology, 211(2), 373-389. doi:10.1083/jcb.201504062

Robida, A. M., \& Kerppola, T. K. (2009). Bimolecular fluorescence complementation analysis of inducible protein interactions: effects of factors affecting protein folding on fluorescent protein fragment association. J Mol Biol, 394(3), 391-409. doi:10.1016/j.jmb.2009.08.069

Rose, R. H., Briddon, S. J., \& Holliday, N. D. (2010). Bimolecular fluorescence complementation: lighting up seven transmembrane domain receptor signalling networks. Br J Pharmacol, 159(4), 738-750. doi:10.1111/j.1476-5381.2009.00480.x

Rudler, D. L., Hughes, L. A., Perks, K. L., Richman, T. R., Kuznetsova, I., Ermer, J. A., . . . Hool, L. C. (2019). Fidelity of translation initiation is required for coordinated respiratory complex assembly. Science advances, 5(12), eaay2118.

Saxton, W. M., \& Hollenbeck, P. J. (2012). The axonal transport of mitochondria. J Cell Sci, 125(Pt 9), 2095-2104. doi:10.1242/jcs.053850

Sheng, Z. H. (2017). The Interplay of Axonal Energy Homeostasis and Mitochondrial Trafficking and Anchoring. Trends Cell Biol, 27(6), 403-416. doi:10.1016/j.tcb.2017.01.005

Shigeoka, T., Jung, H., Jung, J., Turner-Bridger, B., Ohk, J., Lin, J. Q., . . Holt, C. E. (2016). Dynamic Axonal Translation in Developing and Mature Visual Circuits. Cell, 166(1), 181-192. doi:10.1016/j.cell.2016.05.029

Shigeoka, T., Koppers, M., Wong, H. H., Lin, J. Q., Cagnetta, R., Dwivedy, A., . . Holt, C. E. (2019). OnSite Ribosome Remodeling by Locally Synthesized Ribosomal Proteins in Axons. Cell Rep, 29(11), 3605-3619 e3610. doi:10.1016/j.celrep.2019.11.025

Smits, P., Smeitink, J., \& van den Heuvel, L. (2010). Mitochondrial Translation and Beyond: Processes Implicated in Combined Oxidative Phosphorylation Deficiencies. Journal of Biomedicine and Biotechnology, 2010, 737385. doi:10.1155/2010/737385

Spillane, M., Ketschek, A., Merianda, T. T., Twiss, J. L., \& Gallo, G. (2013). Mitochondria coordinate sites of axon branching through localized intra-axonal protein synthesis. Cell Rep, 5(6), 15641575. doi:10.1016/j.celrep.2013.11.022

Taylor, A. M., Blurton-Jones, M., Rhee, S. W., Cribbs, D. H., Cotman, C. W., \& Jeon, N. L. (2005). A 
microfluidic culture platform for CNS axonal injury, regeneration and transport. Nat Methods, 2(8), 599-605. doi:10.1038/nmeth777

Vaarmann, A., Mandel, M., Zeb, A., Wareski, P., Liiv, J., Kuum, M., . . Kaasik, A. (2016). Mitochondrial biogenesis is required for axonal growth. Development, 143(11), 1981-1992. doi:10.1242/dev.128926

Wang, W., Rai, A., Hur, E. M., Smilansky, Z., Chang, K. T., \& Min, K. T. (2016). DSCR1 is required for both axonal growth cone extension and steering. J Cell Biol, 213(4), 451-462. doi:10.1083/jcb.201510107

Wobbrock, J. O., Findlater, L., Gergle, D., \& Higgins, J. J. (2011). The aligned rank transform for nonparametric factorial analyses using only anova procedures. Paper presented at the Proceedings of the SIGCHI conference on human factors in computing systems.

Yoon, B. C., Jung, H., Dwivedy, A., O'Hare, C. M., Zivraj, K. H., \& Holt, C. E. (2012). Local translation of extranuclear lamin B promotes axon maintenance. Cell, 148(4), 752-764. doi:10.1016/j.cell.2011.11.064

Zhou, B., Yu, P., Lin, M. Y., Sun, T., Chen, Y., \& Sheng, Z. H. (2016). Facilitation of axon regeneration by enhancing mitochondrial transport and rescuing energy deficits. J Cell Biol, 214(1), 103- 
bioRxiv preprint doi: $\mathrm{https}$ //doi.org/10.1101/2021.01.26.428248; this version posted January 26, 2021. The copyright holder for this preprint (which was not certified by peer review) is the author/funder, who has granted bioRxiv a license to display the preprint in perpetuity. It is made available under aCC-BY 4.0 International license.

Figure 1. Lee, Park et al

A

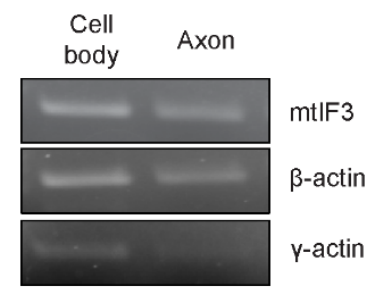

B

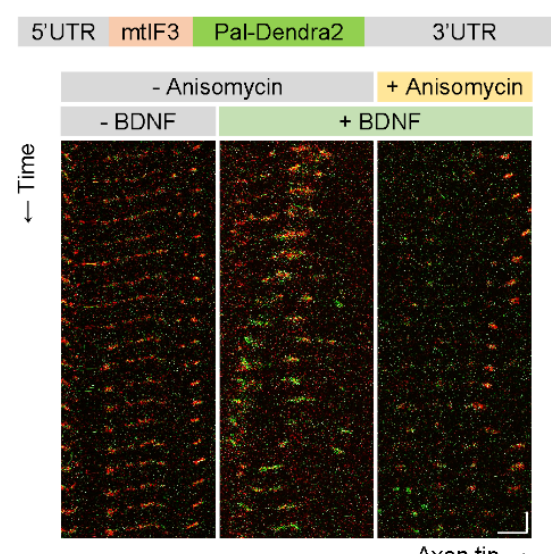

D

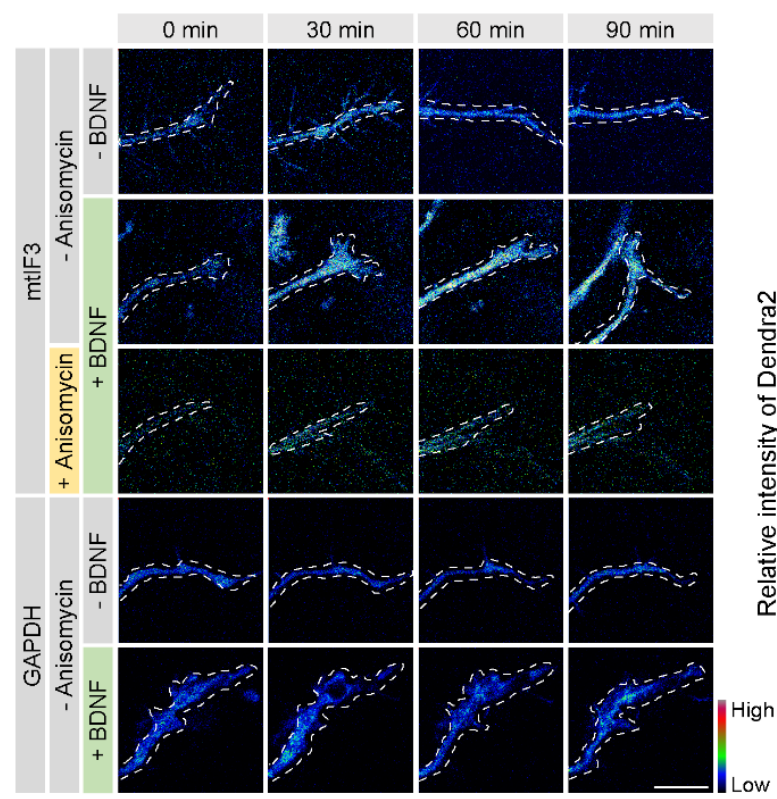

C

$\rightarrow-5^{\prime} U T R_{\text {mitF }}-$ mtIF3-Dendra2-3'UTR $_{\text {miF3 }}$

$\rightarrow-5^{\prime} U T R_{\text {mitF }}$-mtIF3-Dendra2-3'UTR mitF $3+B D N F$

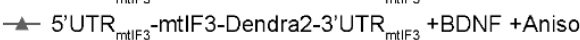

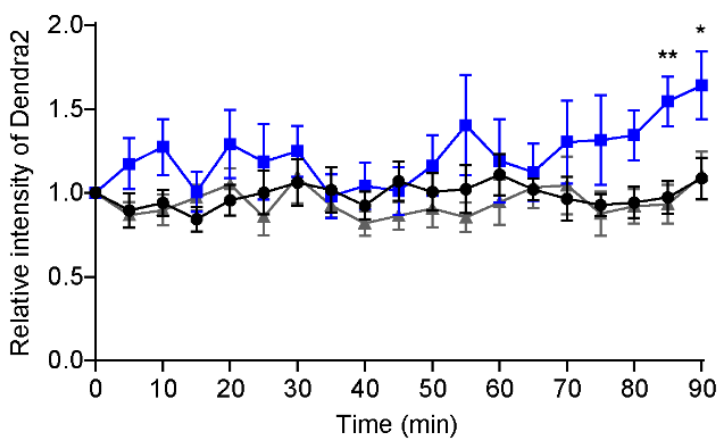

E

$\rightarrow-5^{\prime} U T R_{\text {mtF } 3}$-Dendra2-3'UTR mtF3

- 5'UTR ${ }_{\text {mtF }}$-Dendra2-3'UTR mtF3 + BDNF

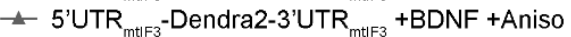

$\rightarrow$ 5'UTR GAPDH -Dendra2-3'UTR

$\rightarrow$ 5'UTR GAPDH -Dendra2-3'UTRR ${ }_{\text {GAPDH }}+$ BDNF

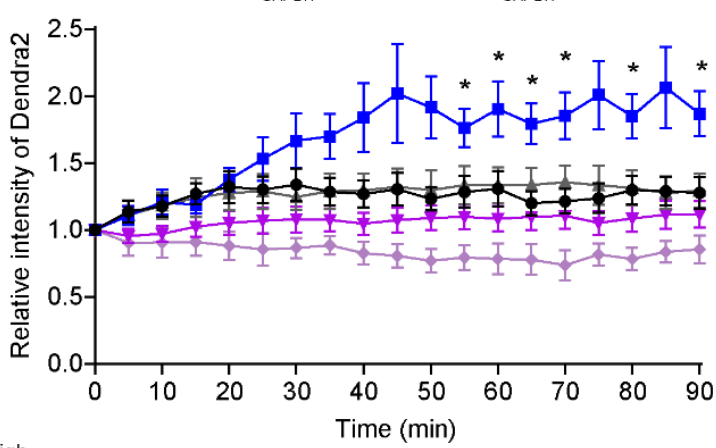

534 Figure 1. BDNF induces local protein synthesis of mtIF3 in the axon growth cone.

535 (A) mtIF3 mRNAs were detected in both cell bodies and axons of primary hippocampal neurons at 
536 DIV4. RNA samples were purified from the isolated lysates of cell bodies and axons. RT-PCR was

537 performed using each pair of gene-specific primers. (B) Kymographs of newly synthesized mtIF3-

538 Dendra2 fusion proteins in axons. Primary hippocampal neurons were transfected with an expression

539 vector for the photoconvertible mtIF3-Dendra2 protein at DIV3, and the fluorescent intensity of

540 mtIF3-Dendra2 fusion was analyzed at DIV4 (horizontal scale bar, $5 \mu \mathrm{m}$; vertical scale bar, 5

541 minutes). The existing mtIF3-Dendra2 was first photoconverted from green to red over the $20 \mu \mathrm{m}$

542 path from the axon tip. Images were then taken at 5-minute intervals for 90 minutes. Where indicated,

543 BDNF $(30 \mathrm{ng} / \mathrm{ml})$ and anisomycin $(20 \mu \mathrm{M})$ were added at a 0-minute timepoint. (C) Quantification of

544 newly synthesized mtIF3-Dendra2 proteins. The green fluorescence was measured from mitochondria

545 at the very end of the axonal tip. The relative intensity at each time point was calculated by

546 normalizing to that at a 0 -minute timepoint. Data represent mean $\pm \operatorname{SEM}(\mathrm{N}=6-8$ axons from 3

547 independent experiments). ${ }^{*} P<0.05, * * P<0.01$, as determined by two-way repeated-measures

548 ANOVA with Dunnett's multiple comparisons test. (D) Pseudo-color images of locally synthesized

549 Dendra2 reporter in the axonal tip. Primary hippocampal neurons were transfected with an expression

550 vector for the photoconvertible Dendra2 reporter harboring the indicated UTRs at DIV3. The newly

551 synthesized Dendra2 reporter was analyzed at DIV4 (scale bar, $10 \mu \mathrm{m}$ ) similarly as in panel B. (E)

552 Quantification of newly synthesized Dendra2 reporters in the axonal tip after drug treatment. Data

553 represent mean $\pm \operatorname{SEM}(\mathrm{N}=6-10$ axons from 3 independent experiments). $* P<0.05$, as determined

554 by two-way repeated-measures ANOVA with Dunnett's multiple comparisons test. 
bioRxiv preprint doi: https://doi. org/10.1101/2021 01 26.428248. this version posted January 26, 2021. The copyright holder for this preprint (which was not certified by peer review) is the author/funder, who has granted bioRxiv a license to display the preprint in perpetuity. It is made available under aCC-BY 4.0 International license.

Figure. 2. Lee, Park et al

A
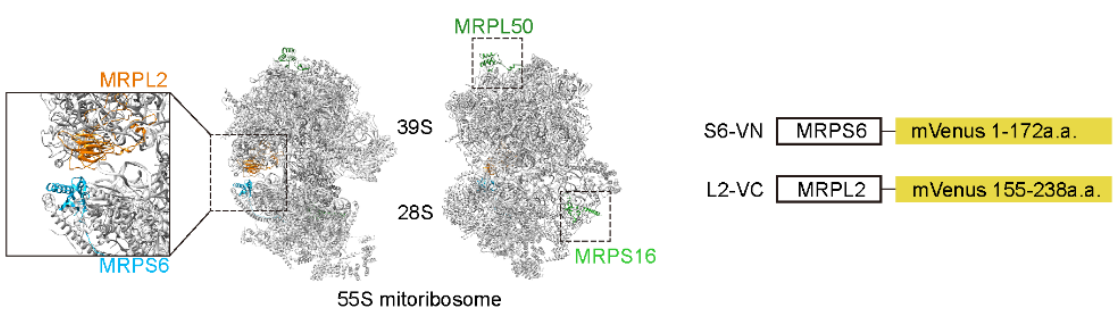

B

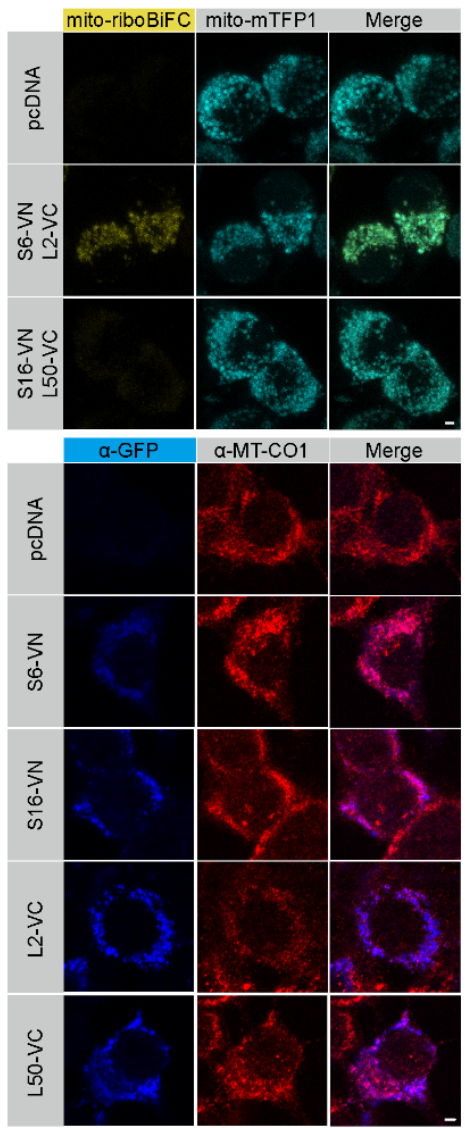

C
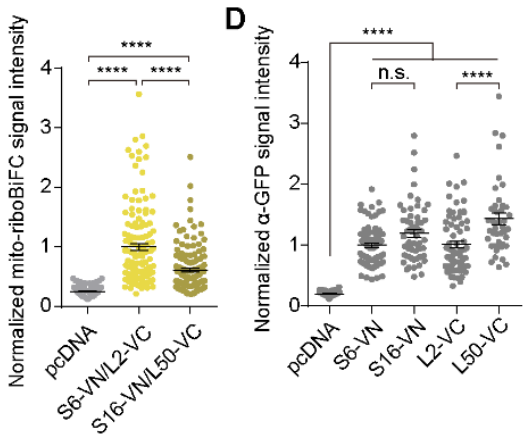

$\mathrm{E}$
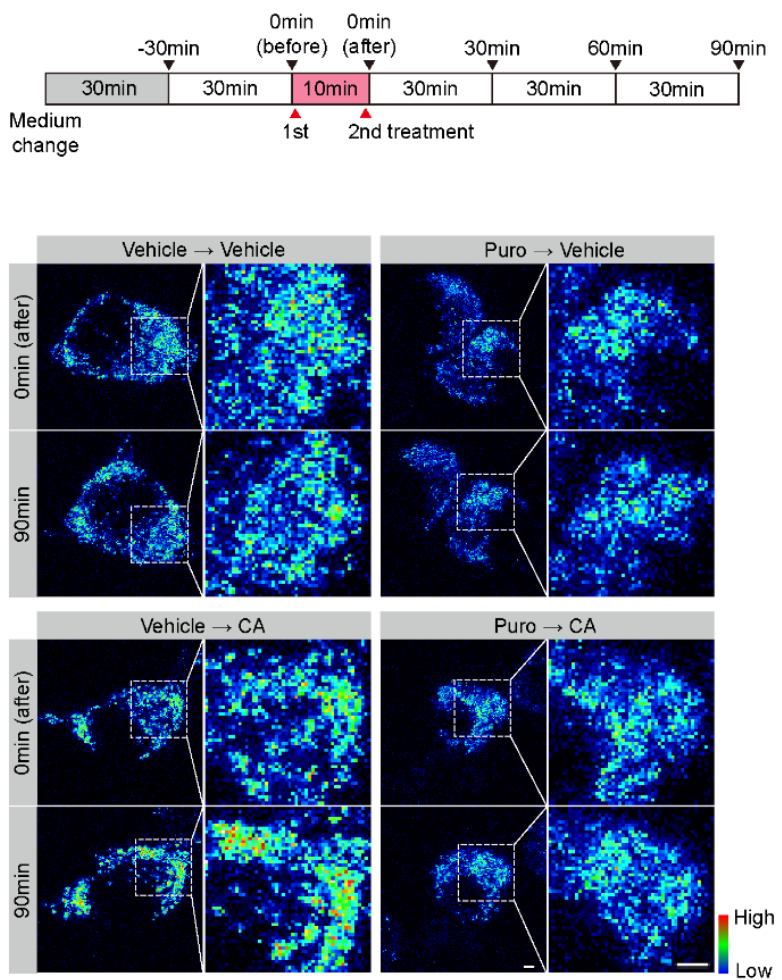

F

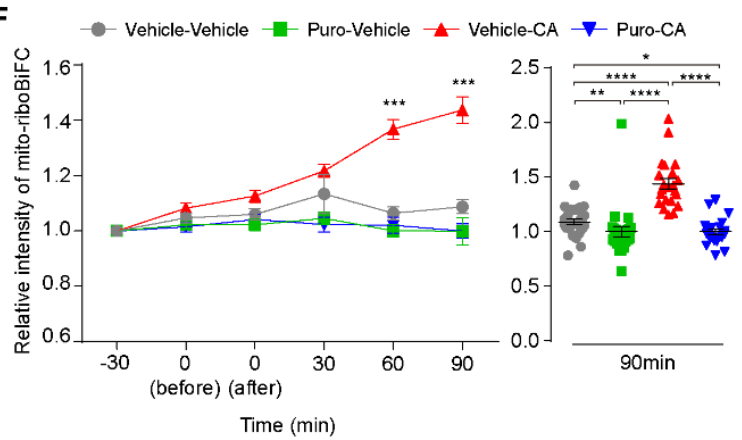

Figure 2. Mito-riboBiFC detects translation-dependent assembly of mitoribosomes.

558 (A) Schematic design of mito-riboBiFC. Mitochondrial ribosomal proteins MRPL2 and MRPS6 were used as a BiFC pair for the mito-riboBiFC and illustrated with porcine 55S mitoribosome cryo-EM 
structure (Greber et al., 2015). MRPS16 and MRPL50 served as a negative control. (B)

561 Representative images of mito-riboBiFC (top) and anti-GFP antibody staining (bottom) in Neuro2A

562 cells. Mito-mTFP1 and MT-CO1 were used as mitochondrial markers (scale bar, $2 \mu \mathrm{m}) .(\mathbf{C})$

563 Quantification of the fluorescent mito-riboBiFC signals in panel B. Data represent mean $\pm \mathrm{SEM}(\mathrm{N}=$ 100-143 cells from 3 independent experiments). $* * * * P<0.0001$, as determined by aligned ranks transformation ANOVA with Wilcoxon rank-sum test. (D) Quantification of At-GFP signal intensity in panel B. Data represent mean \pm SEM $(\mathrm{N}=41-76$ cells from 3 independent experiments). n.s., not significant; $* * * * P<0.0001$, as determined by aligned ranks transformation ANOVA with Wilcoxon rank-sum test. (E) Pseudo-color images of mito-riboBiFC after sequential treatment of puromycin (Puro) and chloramphenicol (CA) (scale bar, $2 \mu \mathrm{m}$ ). (F) Quantification of the mito-riboBiFC signals in panel E. Line plot shows the intensity changes of mito-riboBiFC. A Vehicle-CA group was compared with other groups at each time point for the statistical test (left panel). Dot plot displays the relative intensity of the mito-riboBiFC 90 minutes after CA treatment $\left(1^{\text {st }}\right.$ vehicle, water; $2^{\text {nd }}$ vehicle, ethanol). Data represent mean \pm SEM $(\mathrm{N}=22-26$ cells from 3 independent experiments). Aligned ranks transformation ANOVA detected significant interaction effects of Puro and CA on the mitoriboBiFC intensity at the 90 -minute time point $(P<0.0001) . * * P<0.01, * * * * P<0.0001$, as 
Figure 3. Lee, Park et al

A

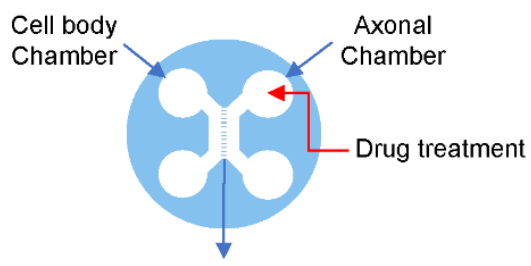

Microgroove $(150 \mu \mathrm{m})$

C

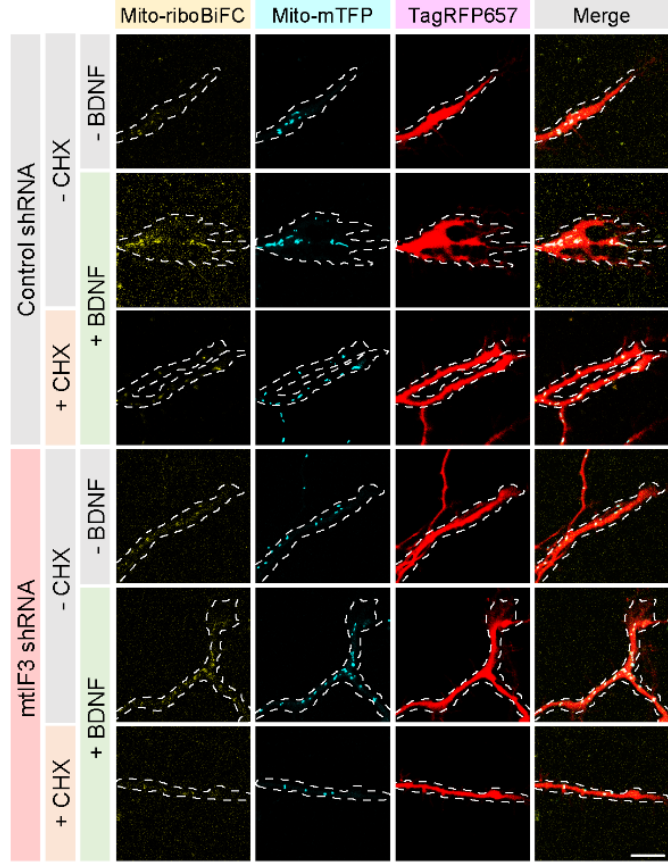

D

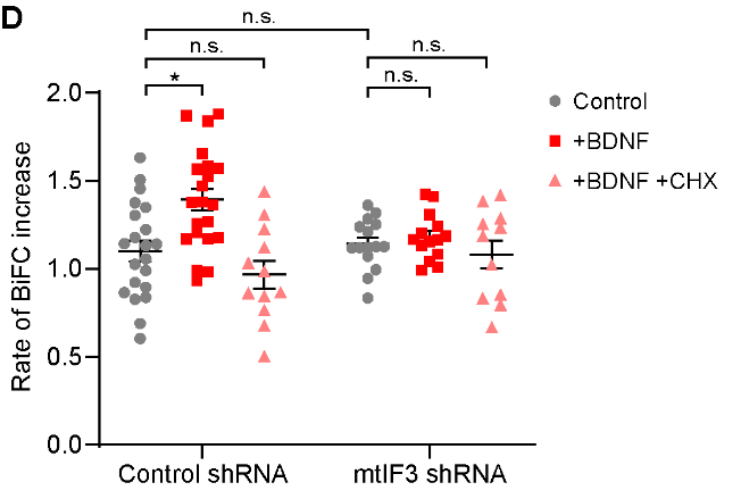

B

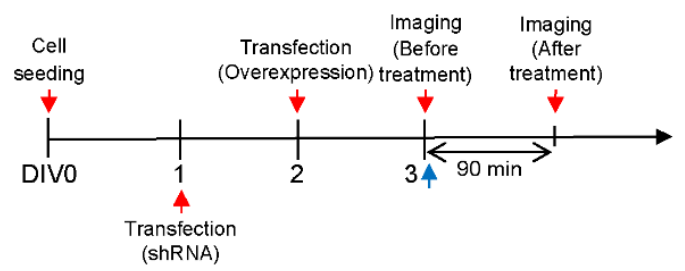

E

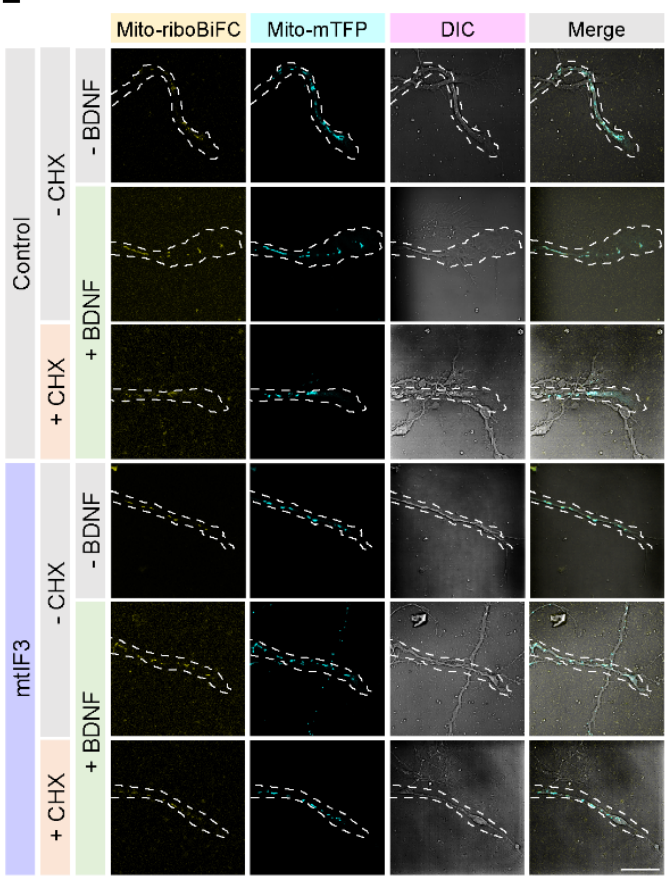

$\mathbf{F}$

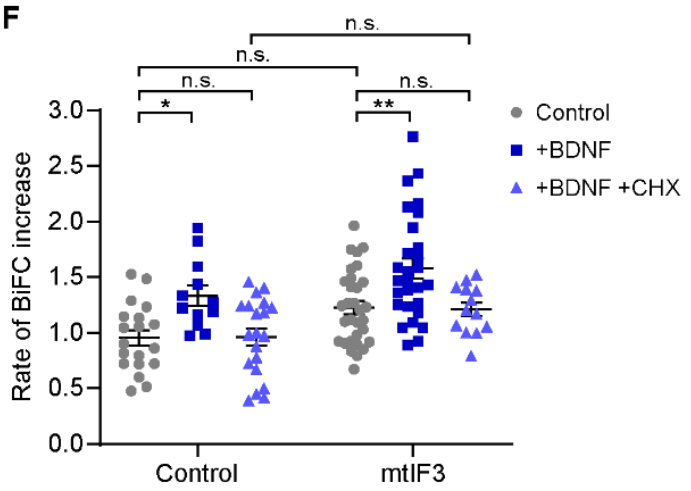

579 Figure 3. Local protein synthesis of mtIF3 is necessary for mitochondrial translation in the axonal growth cone.

581 (A) Schematic illustration of a microfluidic device. Primary hippocampal neurons were seeded into 
582 the cell body chamber. Growing axons reached the other side of the device through the microgroove.

583 To locally stimulate axons, drugs were treated to the axonal chamber. (B) Timeline for mito-riboBiFC

584 experiments. After cell seeding, shRNA and overexpression vectors were transfected at DIV2 and

585 DIV3, respectively. Images were sequentially taken before and after drug treatment. (C, D)

586 Visualization of mitochondrial translation in mtIF3-depleted axon growth cones by mito-riboBiFC.

587 Mitochondria were marked by mitochondria-targeted mTFP1. Transfection of shRNA was confirmed

588 by TagRFP657 expression (scale bar, $20 \mu \mathrm{m}$ ). Mito-riboBiFC was quantified, and the rate of BiFC

589 increase upon drug treatment was measured. Five mitochondria per axon were analyzed. Data

590 represent mean $\pm \operatorname{SEM}(\mathrm{N}=11-21$ axons from 3 independent experiments $)$. Aligned ranks

591 transformation ANOVA detected significant interaction effects of mtIIF3 depletion and BDNF on the

592 BiFC rate $(P=0.0180)$. n.s., not significant; $* P<0.05$, as determined by Wilcoxon rank-sum test

593 (BDNF) or two-way ANOVA with Tukey's multiple comparisons test (BDNF+CHX). (E, F)

594 Representative images of mito-riboBiFC in mtIF3-overexpressing axon growth cones (scale bar, 20

$595 \mu \mathrm{m})$. Mito-riboBiFC signals were analyzed before and after the CA treatment. Five mitochondria per

596 axon were analyzed. Data represent mean \pm SEM $(N=12-31$ axons from 4 independent

597 experiments). Two-way ANOVA detected no significant interaction effects of mtIF3 overexpression

598 and drug treatments. n.s., not significant; ${ }^{*} P<0.05$, ${ }^{*} P<0.01$, as determined by Tukey's multiple

599 comparisons test.

600 
A

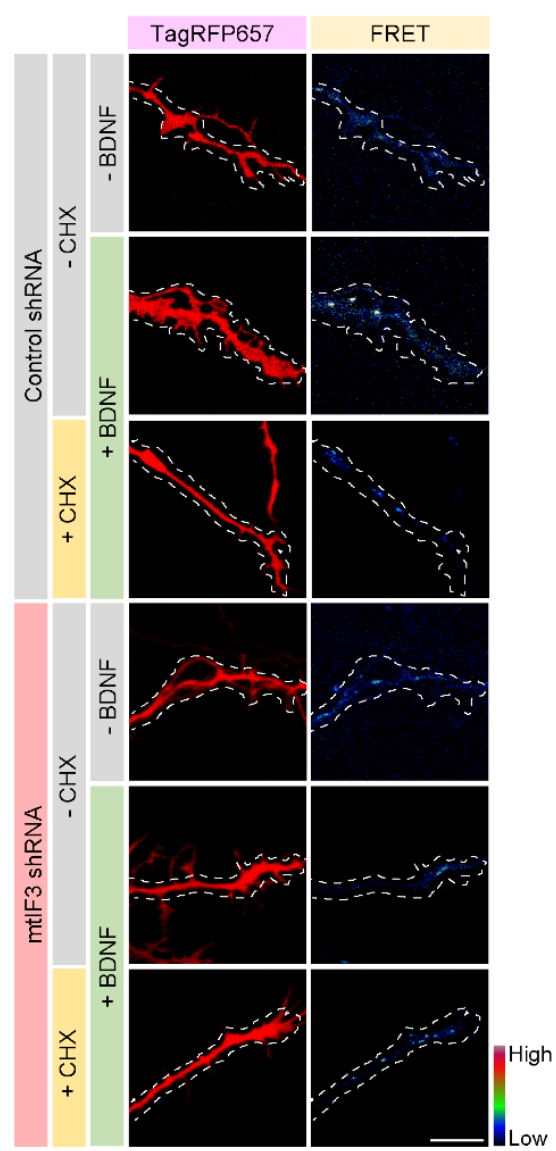

B

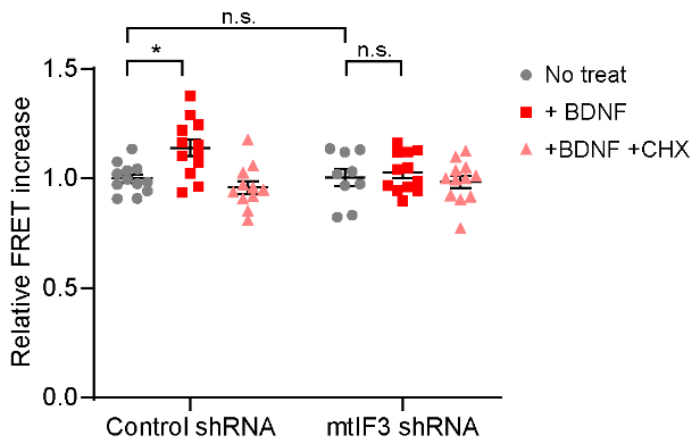

C

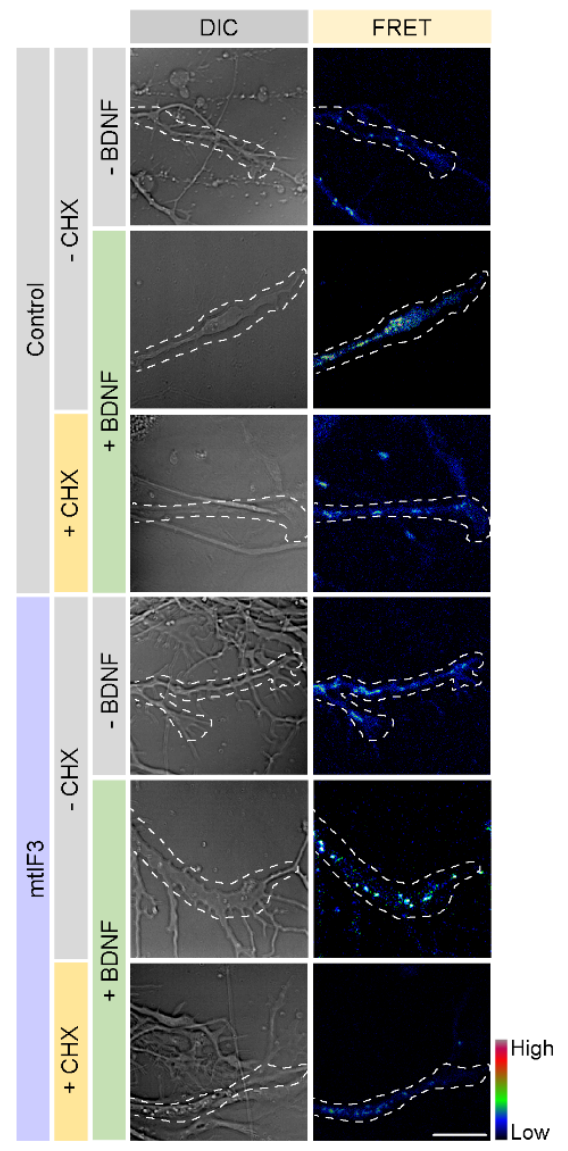

D

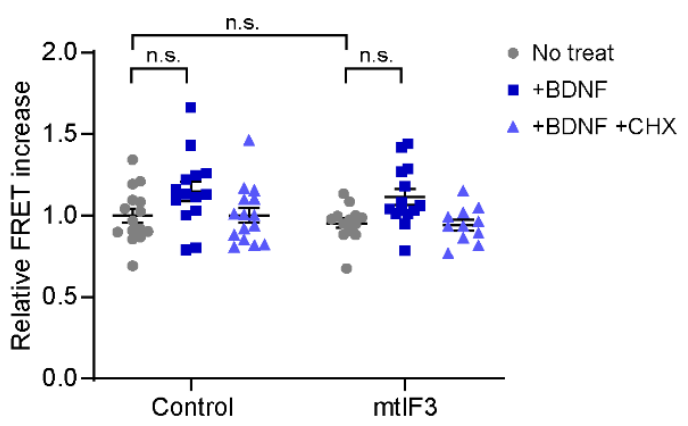

604 (A, C) ATP levels in axonal mitochondria were measured using genetically encoded ATP indicator 
mito-ATeam1.03. FRET signals were shown in the pseudo-color image. Expression of shRNA was confirmed by TagRFP657 expression (scale bar, $10 \mu \mathrm{m}$ ). (B, D) Quantification of the relative FRET

607 intensity in mtIF3-depleted or mtIF3-overexpressing axons. FRET signals were measured by

608 comparing the ratio before and after chemical treatments. Five mitochondria per axon were analyzed.

609 Data represent mean \pm SEM $(\mathrm{N}=9-15$ axons from 4-5 independent experiments). Two-way

610 ANOVA detected significant effects of BDNF, but not of BDNF+CHX, on the FRET signals $(P=$

6110.0124 in panel B; $P=0.0020$ in panel D). n.s., not significant; $* P<0.05$, as determined by Tukey's

612 multiple comparisons test.

613 
A

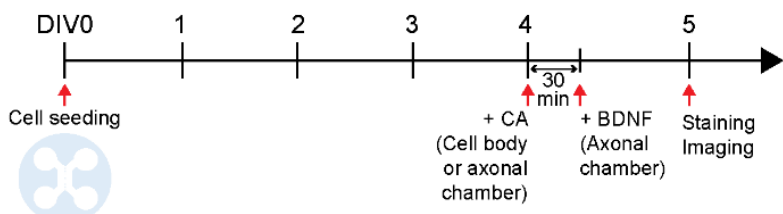

B

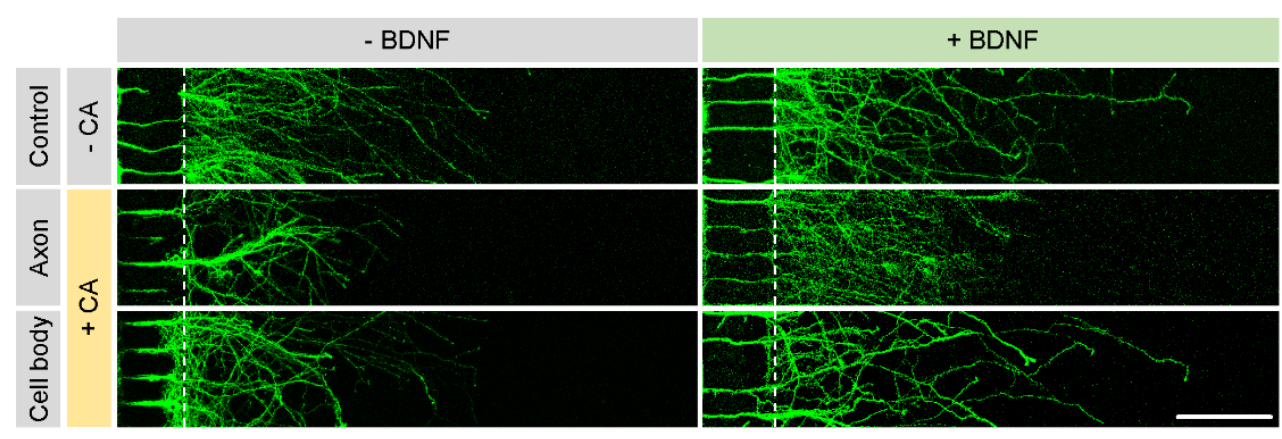

C

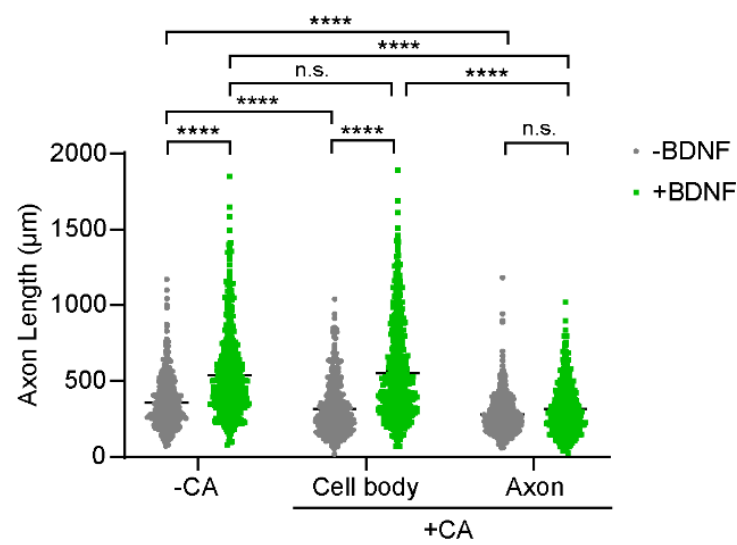

D
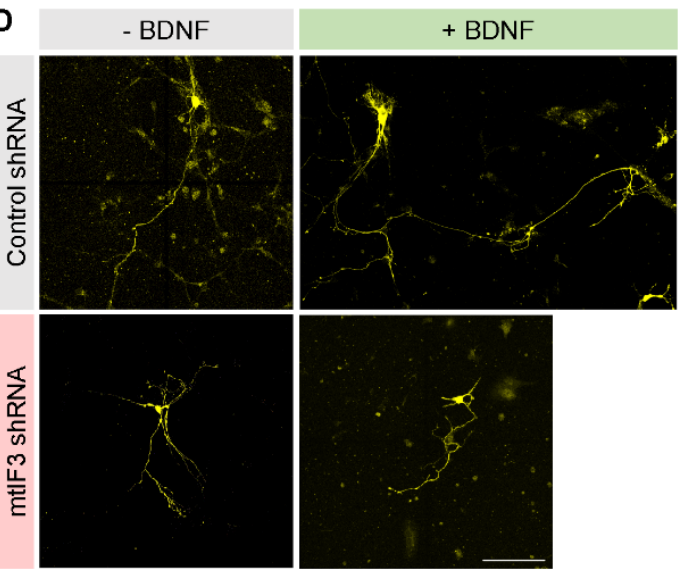

\section{E}

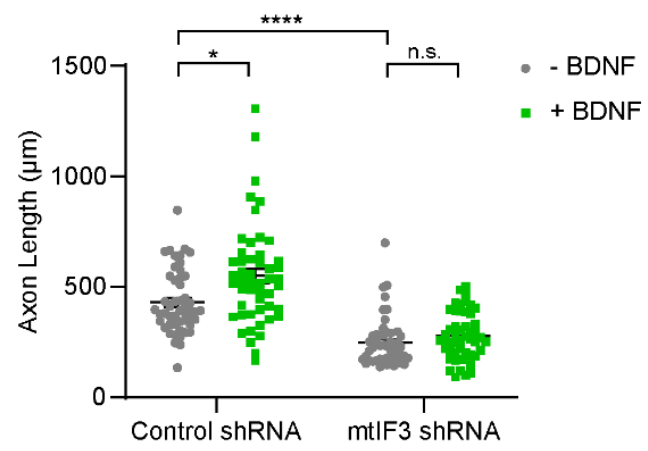

617 (A) Timeline for the experiment. Primary hippocampal neurons were cultured on a microfluidic 
618 device. At DIV4, chloramphenicol (CA) was added to either the cell body or axonal chamber. After

61930 minutes, BDNF was added to the axonal chamber. Neurons were stained and imaged at DIV5. (B)

620 Representative images of axons. Axons were marked by Tau-1 immunostaining (scale bar, $200 \mu \mathrm{m}$ ).

621 (C) The axonal length was measured from the exit border of microgrooves (dotted lines). Data

622 represent mean $\pm \operatorname{SEM}(\mathrm{N}=422-745$ axons from 4-5 independent experiments). n.s., not significant; **** $P<0.0001$, as determined by Aligned ranks transformation ANOVA with Wilcoxon rank-sum test. (D) Representative images of mtIF3-depleted or BDNF-treated primary hippocampal neurons. mtIF3 depletion impaired the extension of the axon growth cone. Hippocampal neurons were first transfected with mtIF3 shRNA and then treated with BDNF at DIV3. The axonal length was measured at DIV5 (scale bar, $100 \mu \mathrm{m}$ ). (E) quantification of axon length in panel D. Data represent mean $\pm \mathrm{SEM}(\mathrm{N}=50$ neurons from 5 independent experiments). Aligned ranks transformation ANOVA detected significant interaction effects of mtIIF3 depletion and BDNF on the axon length $(P$ 
bioRxiv preprint doi: https://doi.org/10.1101/2021.0126.428248; this version posted January 26, 2021. The copyright holder for this preprint (which was not certified by peer review) is the author/funder, who has granted bioRxiv a license to display the preprint in perpetuity. It is made available under aCC-BY 4.0 International license.

\section{Supplementary figure legend}

Figure 1-figure supplement 1. Lee, Park et al

A

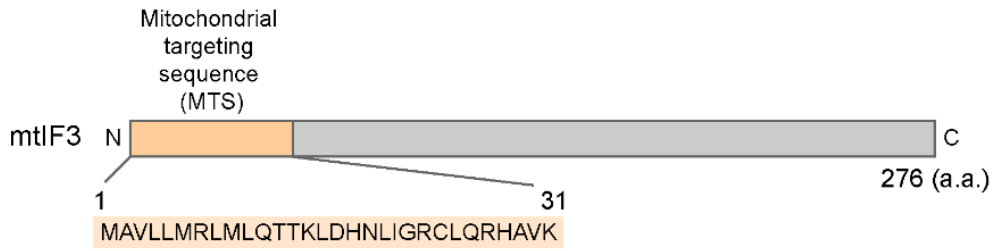

B

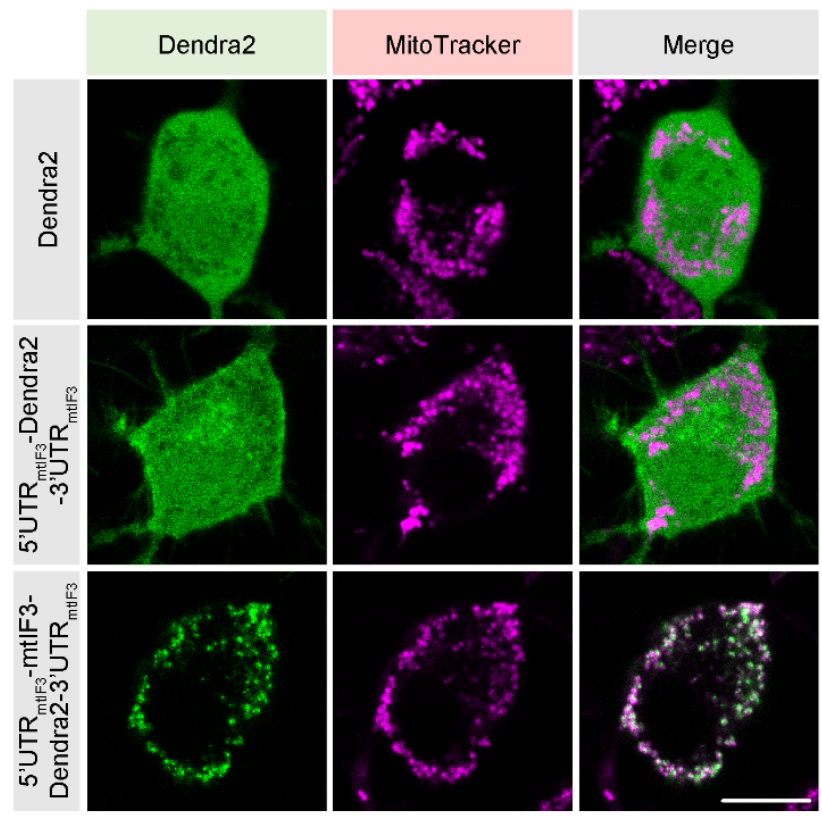

634 Figure 1-figure supplement 1. mtIF3-Dendra2 is localized to mitochondria.

635 (A) Schematic illustration of mtIF3 coding sequence. mtIF3 has mitochondrial targeting sequence in N- 
bioRxiv preprint doi: https://doi.org/10.1101/2021.01.26.428248; this version posted January 26, 2021. The copyright holder for this preprint (which was not certified by peer review) is the author/funder, who has granted bioRxiv a license to display the preprint in perpetuity. It is made available under aCC-BY 4.0 International license.

636 terminal domain (131 a.a.) (Koc \& Spremulli, 2002). (B) Neuro2A cells were transfected with Dendra2

637 vectors. Mitochondria were labeled with MitoTracker deep red dye. CDS of mtIF3 led to mitochondrial

638 localization of Dendra2 (scale bar, $10 \mu \mathrm{m}$ ).

639 

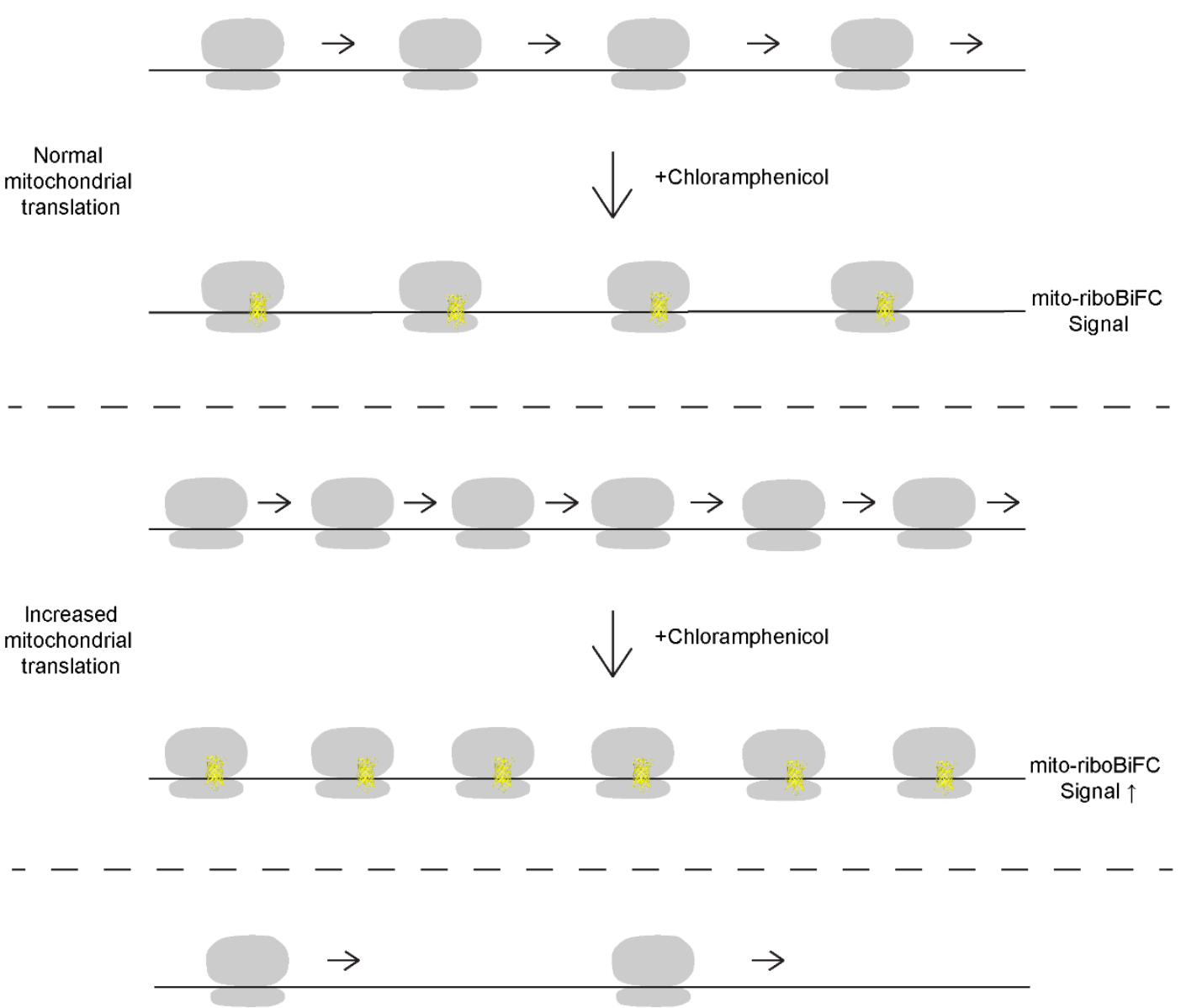

Decreased mitochondrial translation

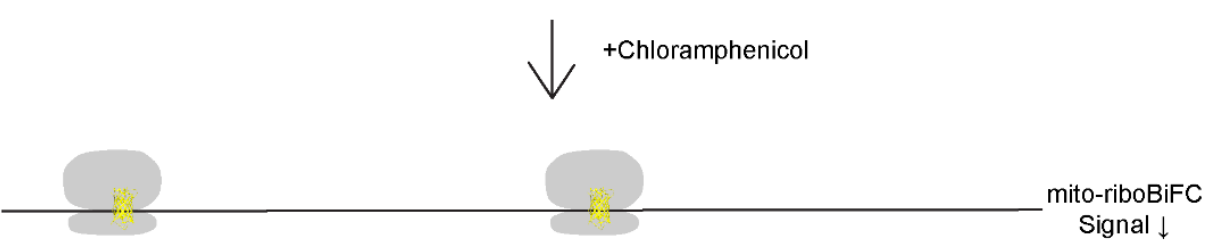

641 Figure 2-figure supplement 1. Schematic illustration of mito-riboBiFC analysis.

642 Translating ribosomal complex exhibits active dynamics. During the elongation, ribosomal subunits consistently rotate, which results in low intensity mito-riboBiFC. To freeze translating mitochondrial 
644 ribosomes, we treated chloramphenicol that inhibits the formation of peptide bond. Non-rotated

645 ribosomal complex is expected to show high BiFC signal. 90 minutes after the treatment of

646 chloramphenicol, we compared the intensity of mito-riboBiFC before and after chloramphenicol

647 treatment. Because highly translating mRNA binds to more ribosomes, we could detect higher signal

648 increase in actively translating mRNA.

649 
A

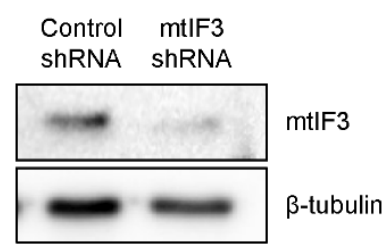

C

Control mtIF3

3xFLAG 3xFLAG
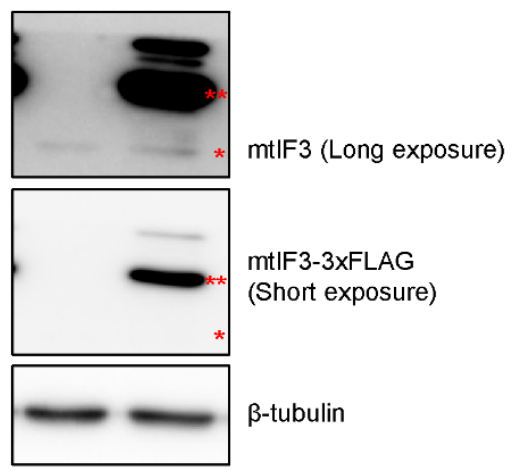

mIIF3-3xFLAG

(Short exposure)

ß-tubulin
B

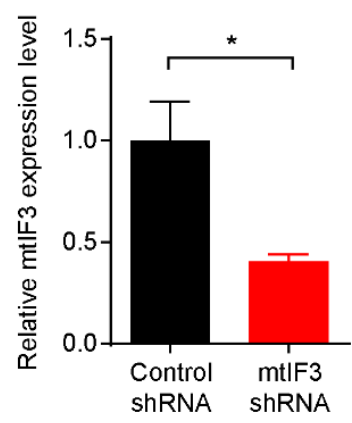

D

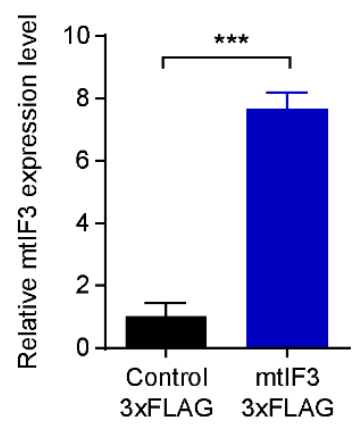

$E$

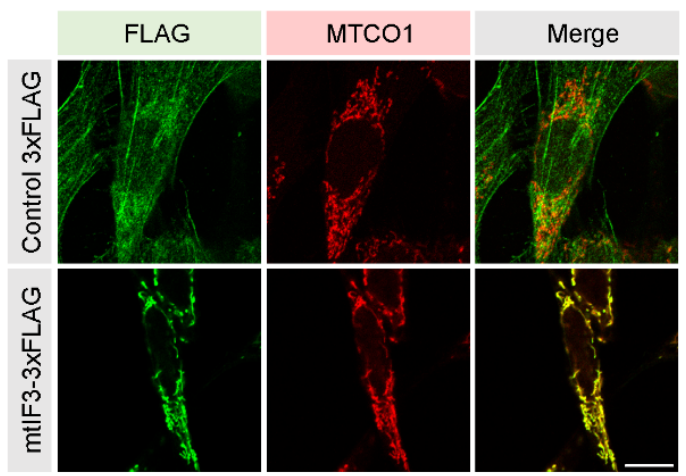

651 Figure 3-figure supplement 1. Modulation of mtIF3 expression level.

652 (A, B) mtIF3 level was reduced by RNA interference with shRNA. By performing western blot, we

653 verified the successful reduction of mtiF3 48 hours after incubation of shRNA in NIH/3T3 cells. The 
654 level of mtIF3 was decreased about 50\% compared with control group. The mtIF3 expression level was

655 normalized to $\beta$-tubulin level. $\mathrm{N}=5$ independent experiments. (C, D) mtIF3 was upregulated by 656 overexpression vector in NIH/3T3 cells. Overexpression vectors contain tandems of FLAG epitope tag.

657 Western blot results showed the expression of mtIF3 overexpression vector. Single red asterisk indicates 658 endogenous mtIF3 and double red asterisk indicates exogenous mtIF3 that is conjugated with FLAG 659 tag. The level of overexpressed mtIF3 was about 8 times higher than endogenous mtIF3 level. $\mathrm{N}=3$ 660 independent experiments. (E) Immunofluorescence images demonstrating the localization of 661 overexpressed mtIF3 to mitochondria in NIH/3T3 cells. FLAG staining detected the transfected 662 overexpression or control vectors. Mitochondria were marked by MTCO1 staining (scale bar, $10 \mu \mathrm{m}$ ).

663 The values were presented as mean \pm SEM and statistical significance was analyzed by unpaired t-test. 664 $* P<0.05, * * * P<0.001$. 


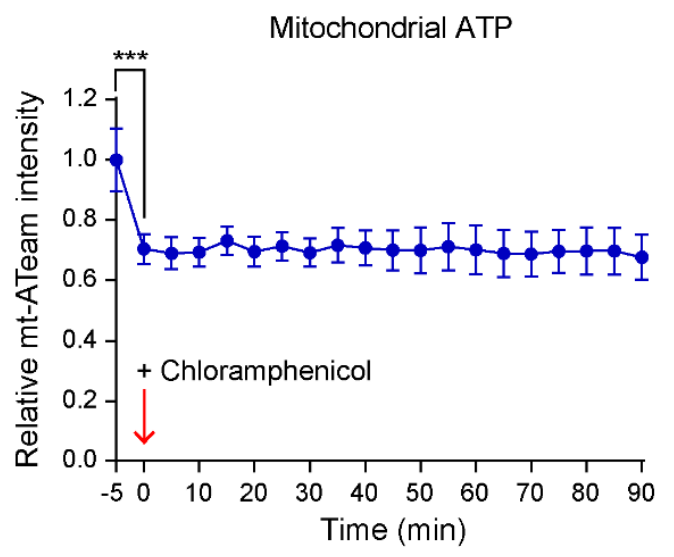

669 Treatment of chloramphenicol caused the rapid decrease of FRET intensity. Primary hippocampal 
670 neurons were transfected with Mt-ATeam1.03 at DIV2 and images were taken every 5 minutes for 90

671 minutes at DIV3. The values are presented as mean \pm SEM and statistical significance was tested

672 between 5 minutes before treatment and 0 minute after treatment using paired t-test. $\mathrm{N}=10$ cells from

6733 independent experiments. $* * * P<0.001$.

674 
bioRxiv preprint doi: https://doi.org/10.1101/2021.01 26.428248; this version posted January 26, 2021. The copyright holder for this preprint (which was not certified by peer review) is the author/funder, who has granted bioRxiv a license to display the preprint in perpetuity. It is made available under aCC-BY 4.0 International license.

Figure 5-figure supplement 1. Lee, Park et al
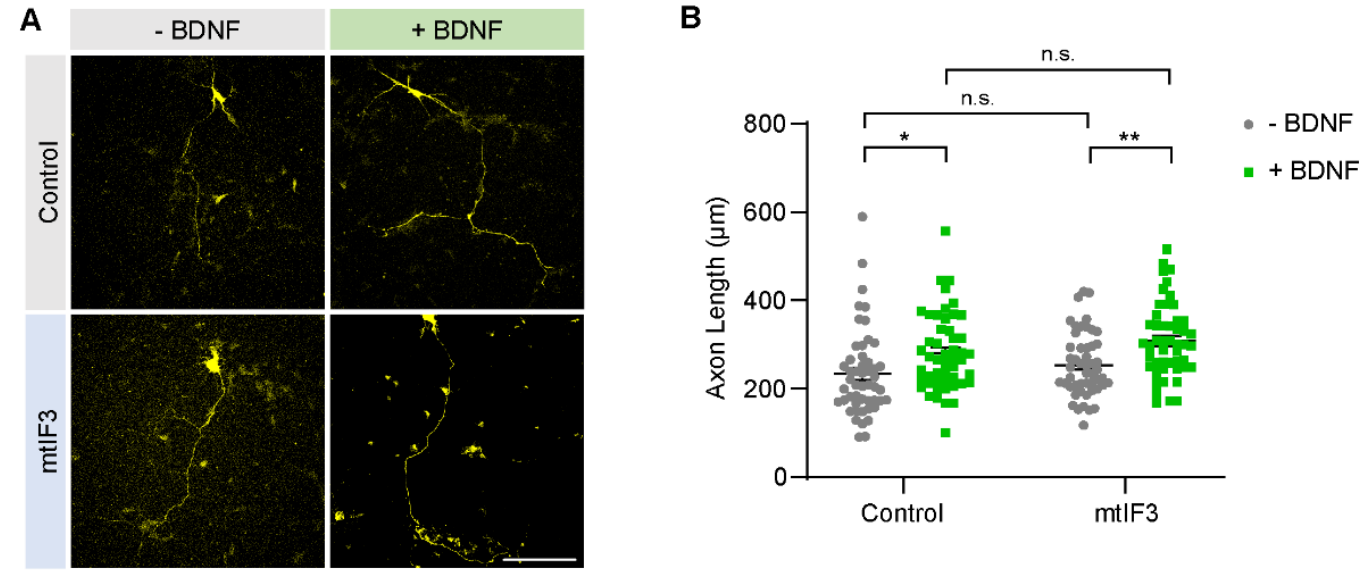

676 Figure 5-figure supplement 1. mtIF3 overexpression is not enough to promote axon extension, but

\section{BDNF treatment still facilitates axon growth.}

678 (A) Representative images of primary neurons expressing mtIF3 overexpression vectors. Hippocampal 
679 neurons were transfected with control and overexpression vectors at DIV2 and BDNF was also treated 680 simultaneously. Images were taken at DIV3. Transfection of overexpression vector was confirmed by 681 TagRFP657 expression. Hippocampal neurons were identified by their morphology (scale bar, $100 \mu \mathrm{m}$ ).

682 (B) Quantification of axonal length. Overexpression of mtIF3 had no additional effect on axon growth, 683 while BDNF treatment still promoted axonal length. Data represent mean $\pm \mathrm{SEM}(\mathrm{N}=50$ neurons from 6845 independent experiments). Aligned ranks transformation ANOVA detected no significant interaction 685 effects of mtIF3 overexpression and drug treatment. n.s., not significant; ${ }^{*} P<0.05, * * P<0.01$, as 686 determined by Wilcoxon rank-sum test. 